DAMTP/94-33

hep-th/9405067

\title{
Quantum Field Theory with Nonzero Minimal Uncertainties in Positions and Momenta
}

\author{
Achim Kempf* \\ Department of Applied Mathematics \& Theoretical Physics \\ University of Cambridge, Cambridge CB3 9EW, U.K. \\ Email: a.kempf@damtp.cambridge.ac.uk
}

\begin{abstract}
A noncommutative geometric generalisation of the quantum field theoretical framework is developed by generalising the Heisenberg commutation relations. There appear nonzero minimal uncertainties in positions and in momenta. As the main result it is shown with the example of a quadratically ultraviolet divergent graph in $\phi^{4}$ theory that nonzero minimal uncertainties in positions do have the power to regularise. These studies are motivated with the ansatz that nonzero minimal uncertainties in positions and in momenta arise from gravity. Algebraic techniques are used that have been developed in the field of quantum groups.
\end{abstract}

*supported by Studienstiftung des Deutschen Volkes, BASF-fellow 


\section{Contents}

\begin{tabular}{lll}
\hline & Introduction & 3
\end{tabular}

$\begin{array}{lll}2 & \text { Quantum mechanics with nonzero minimal uncertainties } & 7\end{array}$

2.1 Quantum mechanics in the Bargmann Fock basis . . . . . . . . . . . 7

2.1 .1 Bargmann Fock space . . . . . . . . . . . . . . . . 7

2.1 .2 Integral kernels . . . . . . . . . . . . . . . . . . . . . . . . 10

2.1 .3 Positions and momenta . . . . . . . . . . . . . . . . . . . 10

2.1.4 Pointwise multiplication . . . . . . . . . . . . . . . . . . 13

2.2 Generalised quantum mechanics in the Bargmann Fock basis . . . . . 14

2.2 .1 Noncommutative Bargmann Fock spaces . . . . . . . . . . . . 14

2.2 .2 Integral kernels . . . . . . . . . . . . . . . . . . . . . . . . . . . . . . . . .

2.2 .3 Positions and momenta . . . . . . . . . . . . . . . . . . . . . . 18

2.2 .4 Pointwise multiplication . . . . . . . . . . . . . . . . . . 24

3 Quantum field theory with nonzero minimal uncertainties $\quad 25$

3.1 Quantum field theory on Bargmann Fock space . . . . . . . . . . . . 30

$3.1 .1 \quad \phi^{4}$-theory . . . . . . . . . . . . . . . . . . . 30

3.1.2 Fields on Bargmann Fock space . . . . . . . . . . . . . . . . . 31

3.1.3 Feynman rules . . . . . . . . . . . . . . . . . . . . . . . . . . . . . . . . . . . . . . . . . 32

3.1.4 Loop integration . . . . . . . . . . . . . . . 34

3.2 Generalised quantum field theory on Bargmann Fock space . . . . . . 34

3.2.1 Generalised $\phi^{4}$ theory . . . . . . . . . . . . . . . . . 36

3.2 .2 Fields on Bargmann Fock space . . . . . . . . . . . . . . . . . 38

3.2.3 $\quad$ Feynman rules . . . . . . . . . . . . . . . . . . . . . . . . . . . 39

3.2 .4 Loop integration . . . . . . . . . . . . . . . . . . . 40

4 On the Poincaré algebra and the origins of minimal uncertainties 42

4.1 Ultraviolet generalisation of the uncertainty relations . . . . . . . . . 42

4.2 Infrared generalisation of the uncertainty relations . . . . . . . . . . . 43

5 Summary and Outlook 48 


\section{Introduction}

Over the past few years there has been considerable progress in the mathematics of noncommutative geometry, and in particular in the field of braided geometry and quantum groups. It has been suggested that the introduction of new 'noncommutative geometric' methods into quantum theory could lead to a deeper understanding of spacetime geometry and thus even to a new connection between quantum theory and gravity.

In this paper we study in fully explicit examples how the quantum field theoretical framework can be generalised for noncommutative position and momentum spaces. Instead of generalising by modifying the linear superposition principle, see e.g. [1], our programme here is to work with generalisations of the Heisenberg commutation relations. It is shown that there then naturally appear nonzero minimal uncertainties, separately in position and in momentum measurements. We demonstrate that the appearance of e.g. a nonzero minimal uncertainty in position has the power to regularise ultraviolet divergencies.

The results of the present paper can thus be viewed as being an approach towards a new regularisation method. This should in itself be interesting. However, studying the effects of nonzero minimal position- or momentum uncertainties in quantum field theory, we do have in mind the idea that gravity could be introduced as the cause of these uncertainties. Let us explain this motivation.

The quantum field theoretical framework, as it is used e.g. for the standard model is certainly very successful from a macroscopic scale down to at least the scale of today's high energy experiments. However, it is clear that the ordinary quantum field theoretical framework cannot describe the physics of the large scales and also not the physics of the very small scales (like the Planck length of $10^{-35} \mathrm{~m}$ ), since in both cases the spacetime structure becomes nontrivial:

In large distances the curvature of spacetime becomes significant and there is on a general curved spacetime no notion of a plane wave. This means that there is a limit to the precision to which momenta can be described. We will make the ansatz that this can be expressed as a (dynamical) nonzero minimal uncertainty in momentum measurements, where we apply the usual definition of the uncertainty in an observable:

$$
(\Delta p)_{|\psi\rangle}^{2}=\left\langle\psi\left|(\mathbf{p}-\langle\psi|\mathbf{p}| \psi\rangle)^{2}\right| \psi\right\rangle
$$

On the other hand, as one tries to measure very small distancies, one needs particles of very high energies, which should eventually also lead to perturbing gravity effects. Therefore we expect there also to be a limit to the precision to which positions can be described. We will make the ansatz that this can be expressed as a (dynamical) nonzero minimal uncertainty in position measurements where we will again apply the usual definition for the uncertainty in an observable.

Although it is quite obvious that in nature positions and momenta can not be measured arbitrarily precisely, even in principle, it is however a nontrivial ansatz to 
assume that this can be expressed as nonzero minimal uncertainties, applying the ordinary quantum theoretical definition of uncertainties in observables.

The ordinary quantum field theoretical framework does not imply a limit to the precision to which positions or momenta could be measured. Mathematically, the ordinary framework deals with arbitrarily small and arbitrarily large scales in position and in momentum space. However the framework does not describe gravity effects that appear in nature at the large and at the very small scales. Instead, the framework allows for infrared and ultraviolet divergencies to occur at the extreme scales.

It appears that the ordinary quantum field theoretical framework suffers from problems, the cause of which can be traced back to quantum mechanics: The (associative) Heisenberg algebra, generated by the hermitean position and momentum operators $\mathbf{x}$ and $\mathbf{p}$, obeying $[\mathbf{x}, \mathbf{p}]=i \hbar$, is represented on the vector space of states. The observables $\mathbf{x}$ and $\mathbf{p}$ have eigenstates, which can be written as $\delta$-functions and plane waves. Now in general, for any eigenstate $|a\rangle$ of an observable $A$ with $A .|a\rangle=a|a\rangle$, the uncertainty in the measurement of $A$ of course vanishes:

$$
(\Delta A)_{|a\rangle}^{2}=\left\langle a\left|(A-\langle a, A . a\rangle)^{2}\right| a\right\rangle=0
$$

The existence of $\mathbf{x}$ - or $\mathbf{p}$ - eigenstates in the representation of the Heisenberg algebra thus implies that the usual quantum theoretical framework allows in principle for the description of arbitrarily precisely measured positions or momenta.

This does not cause many problems on the level of quantum mechanics, apart from the fact that the $\mathbf{x}$ - or $\mathbf{p}$ - eigenstates strictly speaking are not eigenvectors. Dirac $\delta$ 'functions' and plane waves are not square integrable and thus not contained in the Hilbert space. Difficulties with the multiplication of $\delta$-functions are related to that.

The problem is, that the quantum field theoretical framework is built on position space, or equivalently, on momentum space. The notion of points in position or in momentum space, i.e. of particles in position or momentum eigenstates, is thus implemented from the very beginning. Independent of the actual interactions, it is thus the framework itself that pretends that position or momentum measurements of arbitrary precision are possible.

We therefore suggest as a possible cure for this problem to generalise the quantum field theoretical framework such, that it no longer allows for arbitrarily precise measurements of positions or momenta. To this end the quantum fields first have to be redefined on a space alternative to position or momentum space. It is essential to work in a basis other than the position or the momentum eigenbasis because only then can we abandon the very existence of $\mathbf{x}$ - or $\mathbf{p}$ - eigenstates, i.e. the existence of 'points' in position or momentum space.

In a generalised Heisenberg algebra not only the positions and momenta do not commute, now the positions and the momenta may no longer commute even among themselves. While quantisation abandoned the classical phase space, this also abandons the configuration and the momentum space. 
The generalisation parameters $q_{i}$ that control the commutation relations have one special value, say $q_{i}=1$, that corresponds to the ordinary quantum field theoretical framework. The generalised Heisenberg algebra, then involving a length scale, should imply nonzero minimal uncertainties in positions and momenta. Intuitively a nonzero minimal uncertainty e.g. in positions is of course like a short-distance cutoff. It is however nontrivial to show that a nonzero minimal $x$ - uncertainty really leads to the regularisation of otherwise ultraviolet divergent graphs, with the divergency only to be recovered when the generalisation parameters approach $q_{i}=1$.

The finite values of the loop integrations together with the sizes of the minimal uncertainties depend on the generalisation parameters. As we said, ultimately we expect nonzero minimal uncertainties in positions and momenta to be arising from gravity. Thus, the further idea is to try to relate the generalisation parameters (which then have to be dynamical) to gravitational degrees of freedom. In this way gravity effects might actually regularise quantum field theory. To make contact with gravity is beyond the scope of the present paper. We will only sketch a possible ansatz in Section 4.2 .

The programme for our studies is as follows:

First we reformulate the ordinary quantum field theoretical framework such that the quantum fields no longer live on position or momentum space. Instead of the basis of $\delta$-functions or plane waves we will here choose to define them on a basis of Bargmann Fock functions. Other choices are possible, the main objective is, as we said, to achieve independence of the $x$ - eigenbasis and the $p$ - eigenbasis. Up to this point we only reformulate the ordinary formalism, with the fields now living on an alternative space such as Bargmann Fock space, rather than on position or momentum space. The unitary transformations to and from quantum fields that are defined on position or momentum space are given.

Secondly then, we generalise the Heisenberg algebra. In principle we are interested in generalising its commutation relations as far as consistently possible. For explicit calculations Hilbert space representations for the generalised Heisenberg algebras are needed. We postpone the fully general construction of Hilbert space representations. As we will see, large classes of generalised Heisenberg commutation relations can be represented on certain generalised Bargmann Fock spaces. We will here consider only these restricted generalisations of the Heisenberg algebras, since for these we can easily find Hilbert space representations, namely by using quantum group methods.

This strategy of first reformulating the ordinary quantum theoretical formalism in an alternative (e.g. the Bargmann Fock-) basis and then secondly generalising the Heisenberg algebra, we will be following in Section 2 for quantum mechanics and in section 3 for quantum field theory.

The set of generalised commutation relations that we can deal with up to now is, as we will see in Section 4, not yet large enough for an attempt to describe gravity. These restricted generalisations do however already show features that we expect to 
be arising from gravity: With the generalised commutation relations, the position and momentum operators do no longer have eigenfunctions in the representations of the Heisenberg algebra. Instead, there are now nonzero minimal uncertainties in $\mathbf{x}-$ and in $\mathbf{p}$ - measurements. Intuitively one can expect that this implies short distance cutoffs in position and in momentum space. Indeed, working with $\phi^{4}$ theory we will show for the example of a quadratic ultraviolet divergency that the introduction of nonzero minimal uncertainties in positions does indeed have the power to regularise.

The significance of this result is, that it supports the idea that gravity effects can be introduced into quantum field theory in such a way that they actually regularise quantum field theory.

As we said, we will here work only with specific examples of a general approach. With further Hilbert space representations of more generalised Heisenberg algebras it should be possible to straightforwardly follow the same programme to formulate the correspondingly generalised quantum theoretical framework.

The representations that we will use here for our examples of generalised Heisenberg commutation relations are representations on so called $q$ - Bargmann Fock spaces, which have been developed in the context of quantum groups. We do therefore naturally include the case when the commutation relations are preserved by a quantum group of linear quantum canonical transformations. However, let us stress that we are covering a more general case and in particular that we do not have a quantum group of external symmetry. The wave functions will be commutative and we will not be dealing with particles that obey braid statistics. We are using the algebraic techniques that have been developed in the field of quantum groups merely as a powerful tool for the construction of certain Hilbert space representations.

Standard references for the mathematics of quantum groups are [2]-[8]. The $S U_{q}(n)$ symmetric Bargmann Fock spaces that we will be using have been constructed in [9, 10], for questions of uniqueness see also [11, 12]. Using this formalism for nonrelativistic quantum mechanics the study of nonzero minimal uncertainties in $\mathbf{x}$ and $\mathbf{p}$ has been worked out in [13].

There are many other approaches towards the implementation of several kinds of noncommutative geometry into quantum theory or gravity, see e.g. [13-30]. In particular, some of the quantum group symmetric commutation relations that we use here, which were derived in the $R$-matrix approach in [9], had already, as I learned later, appeared in a different approach in 31. However, our generalised Heisenberg algebras with their Bargmann Fock representations, the derivation of the nonzero minimal uncertainties, and our field theoretical studies here, are new. An interesting new alternative approach is [34].

The idea of the existence of a minimal observable length is of course not new, see e.g. [37, 38, and for a recent survey [39]. Interestingly an effective uncertainty relation implying nonzero minimal uncertainties in positions has been derived in the context 
of string theory, where it effectively arises from the string size, see e.g. 41, 42, 43, and references therein. That uncertainty relation is a special case of the uncertainty relation of our framework. This may indicate that our framework could serve as an effective theory of an underlying theory of explicitly non-pointlike particles, such as string theory. The connection between the generalisation parameters and the gravitational degrees of freedom would then of course be quite indirect.

\section{Quantum mechanics with nonzero minimal un- certainties}

In the two sections 2.1 and 2.2 of this chapter we study quantum mechanics and our generalisations of it in a parallel way.

We choose to work in the Bargmann Fock basis of the (Hilbert) space of states. In order to write operators as integral kernels we use a new formula for the integral over two Bargmann Fock functions. This algebraic integral also works in the generalised case. Studying the position and momentum operators we give the unitary transformations that map from the Bargmann Fock basis to the position or the momentum eigenbasis and vice versa. In the generalised case there are no more position or momentum eigenstates and the uncertainty relation implies nonzero minimal uncertainties in positions and momenta. We develop new algebraic techniques which will allow us to generalise the notion of pointwise multiplication to the noncommutative situation. This will be crucial in section 3 for the description of local interactions in quantum field theory.

\subsection{Quantum mechanics in the Bargmann Fock basis}

In the following four subsections we deal with ordinary quantum mechanics. After a brief review of the notion of Bargmann Fock representation we develop some useful new formulas for the transformations from Bargmann Fock space to position and momentum space, and vice versa. We then apply them to the translation of the notion of pointwise multiplication from position space into Bargmann Fock space.

\subsubsection{Bargmann Fock space}

The Heisenberg algebra, generated by the observables $\mathbf{x}$ and $\mathbf{p}$, obeying $[\mathbf{x}, \mathbf{p}]=i \hbar$, is represented on the space of states in which one usually chooses a basis of position or momentum eigenvectors $|x\rangle,|p\rangle$. The states are then written expanded as wave functions $\psi_{x}(x):=\langle x \mid \psi\rangle$ or $\psi_{p}(p):=\langle p \mid \psi\rangle$ and $\mathbf{x}, \mathbf{p}$ act on them as multiplication and differentiation operators $x,-i \hbar \partial_{x}$ or $i \hbar \partial_{p}, p$. The coordinates $\langle x \mid \psi\rangle$ of a state $|\psi\rangle$ in the basis of position eigenvectors are unitarily transformed into its coordinates $\langle p \mid \psi\rangle$ in the basis of momentum eigenvectors using $\langle p \mid x\rangle=(2 \pi \hbar)^{-1 / 2} e^{-i p x / \hbar}$, i.e. by Fourier transformation $\langle p \mid \psi\rangle=\int_{-\infty}^{+\infty} d x\langle p \mid x\rangle\langle x \mid \psi\rangle$. 
It is well known that alternatively one can e.g. choose the basis of eigenvectors of the operator $\bar{\eta} \partial_{\bar{\eta}}$ where:

$$
\bar{\eta}:=\frac{1}{2 L} \mathbf{x}-\frac{i L}{\hbar} \mathbf{p} \quad \text { and } \quad \partial_{\bar{\eta}}:=\frac{1}{2 L} \mathbf{x}+\frac{i L}{\hbar} \mathbf{p}
$$

Here, with $L$ we introduce an arbitrary positive length. One checks that $\bar{\eta}$ and $\partial_{\bar{\eta}}$ indeed obey $\partial_{\bar{\eta}} \bar{\eta}-\bar{\eta} \partial_{\bar{\eta}}=1$, which is of the form of a Leibniz rule. For the Bargmann Fock operator we readily write down the countable set of eigenvectors $\bar{\eta} \partial_{\bar{\eta}}\left|\bar{\eta}^{n}\right\rangle=n\left|\bar{\eta}^{n}\right\rangle$ with $n=0,1,2, \ldots$ which are found to have the scalar product:

$$
\left\langle\bar{\eta}^{n} \mid \bar{\eta}^{m}\right\rangle=n ! \delta_{n, m}
$$

With the definitions $\left|a \bar{\eta}^{n}+b \bar{\eta}^{m}\right\rangle:=\left|a \bar{\eta}^{n}\right\rangle+\left|b \bar{\eta}^{m}\right\rangle$ and $a\left|\bar{\eta}^{n}\right\rangle:=\left|a \bar{\eta}^{n}\right\rangle$, arbitrary states $|\psi\rangle$ can be written as polynomials or power series

$$
|\psi(\bar{\eta})\rangle=\left|\sum_{r=0}^{\infty} \psi_{r} \frac{\bar{\eta}^{r}}{\sqrt{r !}}\right\rangle
$$

The action of the generators $\mathbf{x}$ and $\mathbf{p}$ of the Heisenberg algebra is then obtained from Eqs.11, namely

$$
\mathbf{x}=L\left(\bar{\eta}+\partial_{\bar{\eta}}\right) \quad \mathbf{p}=\frac{i \hbar}{2 L}\left(\bar{\eta}-\partial_{\bar{\eta}}\right)
$$

so that e.g.:

$$
\mathbf{x}|\psi(\bar{\eta})\rangle=L\left|\bar{\eta} \psi(\bar{\eta})+\partial_{\bar{\eta}} \psi(\bar{\eta})\right\rangle
$$

We have

$$
\left\langle x \mid \bar{\eta}^{n}\right\rangle=\sqrt{n !}\left(2 \pi L^{2}\right)^{-1 / 4}\left(x / 2 L-L \partial_{x}\right)^{n} e^{-\frac{1}{4}\left(\frac{x}{L}\right)^{2}}
$$

which are, up to a factor, the Hermite functions. The coordinates $\psi_{n}=\left\langle\bar{\eta}^{n} \mid \psi\right\rangle / \sqrt{n}$ ! of a function $|\psi\rangle$, in the orthonormalised basis of monomials $\bar{\eta}^{n} / \sqrt{n !}$, are unitarily transformed into its coordinates $\psi_{x}(x)=\langle x \mid \psi\rangle$ in the basis of position eigenvectors:

$$
\langle x \mid \psi\rangle=\sum_{n=0}^{\infty}\left\langle x \mid \bar{\eta}^{n}\right\rangle\left\langle\bar{\eta}^{n} \mid \psi\right\rangle(n !)^{-1}
$$

To summarise, we are presently considering three different bases of our space of states. In particular, the set of states $|\psi\rangle$ which yield a square integrable wave function $\psi_{x}(x)=\langle x \mid \psi\rangle$ (and thus also a square integrable $\psi_{p}(p)=\langle p \mid \psi\rangle$ ) forms a Hilbert space $\mathcal{H}$ and these states can also be written as holomorphic, weighted square integrable 'Bargmann Fock functions' $|\psi(\bar{\eta})\rangle$ and vice versa. Recall that the scalar product of two arbitrary states, when given as Bargmann Fock functions, can be calculated e.g. as

$$
\langle\psi(\bar{\eta}) \mid \phi(\bar{\eta})\rangle=\frac{1}{2 \pi i} \int d \eta d \bar{\eta} \overline{\psi(\bar{\eta})} e^{-\bar{\eta} \eta} \phi(\bar{\eta})
$$


where the $\psi(\bar{\eta})$ and $\phi(\bar{\eta})$ on the rhs are now to be read as polynomials or power series in ordinary complex variables rather than in Bargmann Fock operators $\bar{\eta}$.

We should here clarify an important point:

A 'wave function' gives the coefficients of a state in a particular basis. E.g. $\psi(x)=\langle x \mid \psi\rangle$ and $\psi(p)=\langle p \mid \psi\rangle$ are the coefficients of the state $|\psi\rangle$ in the position or momentum eigenbasis, i.e. they are the position or momentum wave functions. However, Bargmann Fock functions $\psi(\bar{\eta})$ are not円 wave functions! $\psi(\bar{\eta})$ is a function, or more precisely, it is a polynomial or power series in the Bargmann Fock operator $\bar{\eta}$ and thus it is itself an operator on the representation space. Applied on the normalised state $|1\rangle$ (defined through $\partial_{\bar{\eta}} \cdot|1\rangle=0$ ) one obtains a state $\psi(\bar{\eta})|1\rangle$ or $|\psi(\bar{\eta})\rangle$. This state is usually also simply denoted by $\psi(\bar{\eta})$. (Thus, in particular the state $|\bar{\eta}\rangle$ is defined as $\bar{\eta} .|1\rangle$ and it is not an eigenstate to the operator $\bar{\eta}$.)

On the other hand, the Bargmann Fock operator $\bar{\eta} \partial_{\bar{\eta}}$ is self-adjoint with the eigenbasis $\left\{\left|(r !)^{-1 / 2} \bar{\eta}^{r}\right\rangle\right\}(r=0,1,2, \ldots)$. Just like the position or momentum wavefunctions $\langle x \mid \psi\rangle$ or $\langle p \mid \psi\rangle$ that we get as the coefficients of a state in the $\mathbf{x}$ or $\mathbf{p}$ - eigenbases $\{|x\rangle\}$ or $\{|p\rangle\}$, we can therefor now also calculate a Bargmann Fock wave function:

$$
\psi_{r}=\left\langle(r !)^{-1 / 2} \bar{\eta}^{r} \mid \psi\right\rangle \quad r=0,1,2, \ldots
$$

Unlike the $\mathbf{x}$ or the $\mathbf{p}$ eigenbases, the eigenbasis of the Bargmann Fock operator $\bar{\eta} \partial_{\bar{\eta}}$ is countable, so that our Bargmann Fock wave functions are actually sequences rather than functions (we are making use of the fact that the Hilbert space of states is separable).

Let us carefully distinguish the Bargmann Fock functions $\psi(\bar{\eta})$ or $|\psi(\bar{\eta})\rangle$, which denote operators on or vectors in the representation space, from the Bargmann Fock wave functions $\psi_{r}$, which are the complex number valued coefficients of a state, when expanded in the eigenbasis of the operator $\bar{\eta} \partial_{\bar{\eta}}$.

This is to be kept in mind when we will be speaking, like in the literature, about Bargmann Fock functions $\psi(\bar{\eta})$ on 'Bargmann Fock space'. It should now be clear that what is meant is not a wave function, but a state, namely the state $\psi(\bar{\eta}) \cdot|1\rangle$.

In our generalised framework we will be dealing with Bargmann Fock functions $\psi\left(\bar{\eta}_{i}\right)$ where e.g. the Bargmann Fock operators $\bar{\eta}_{i}$ no longer commute. Nevertheless, the Bargmann Fock wave functions $\psi_{r}$, i.e. the coefficients of the states expanded in the eigenbasis of $\bar{\eta}_{i} \partial_{\bar{\eta}_{i}}$ remain of course complex number valued, and thus the Bargmann Fock wave functions remain commutative. Further then this will mean that when we generalise e.g. bosonic $\phi^{4}$ - theory, the fields that we sum over in the path integral are strictly commutative. We will see that even in the case when the commutation relations of the Heisenberg algebra are conserved under the action of a quantum group, we will not be dealing with particles that obey braid statistics?

\footnotetext{
${ }^{1} \psi(\bar{\eta})$ is not the coefficient of the state $|\psi\rangle$ in a ' $\bar{\eta}$-eigenbasis'. An $\bar{\eta}$-eigenbasis does not exist. We are also not dealing with the (overcomplete) coherent states, which would be eigenstates of the operator $\partial_{\bar{\eta}}$.

${ }^{2}$ Recall that the 'universal R-matrix' that determines the quasitriangular structure of a quantum
} 


\subsubsection{Integral kernels}

We recall that, using the weighted complex integral Eq.7, operators can also be written as integral kernels: Let $P\left(\bar{\eta}, \partial_{\bar{\eta}}\right)$ be an operator on the Hilbert space, expressed in terms of the Bargmann Fock operators $\bar{\eta}$ and $\partial_{\bar{\eta}}$. (Using Eqs. 3 it could of course also be written in terms of the position and momentum operators.)

The integral kernel $G_{P}\left(\bar{\eta}^{\prime}, \eta\right)$ of $P$ is then a function in the $\bar{\eta}^{\prime}$ and $\eta$, which lives in a primed and an unprimed copy of the function space. Integrating $G_{P}$ over an arbitrary Bargmann Fock function $\psi(\bar{\eta})$ yields the action of the operator $P$ on this Bargmann Fock function:

$$
P\left(\bar{\eta}^{\prime}, \partial_{\bar{\eta}^{\prime}}\right) \psi(\bar{\eta})=\frac{1}{2 \pi i} \int d \bar{\eta} d \eta G_{P}\left(\bar{\eta}^{\prime}, \eta\right) e^{-\bar{\eta} \eta} \psi(\bar{\eta})
$$

The integral kernel of the identity operator is $G_{1}\left(\bar{\eta}^{\prime}, \eta\right)=e^{\bar{\eta}^{\prime} \eta}$ and e.g. the integral kernel of the time evolution operator $U=e^{-i\left(t_{f}-t_{i}\right) \omega \bar{\eta} \partial_{\bar{\eta}}}$ of the harmonic oscillator, is the Greens function:

$$
G_{U}\left(\bar{\eta}^{\prime}, \eta\right)=e^{\bar{\eta}^{\prime} \eta e^{-i \omega\left(t_{f}-t_{i}\right)}}
$$

\subsubsection{Positions and momenta}

The operators $\mathbf{x}$ and $\mathbf{p}$ have (non-normalisable) eigenvectors, namely ' $\delta$ - functions' and plane waves, which are the 'points' in position or momentum space. How do these states read in the Bargmann Fock basis, and in general, how is a wave function that is given on position space transformed into Bargmann Fock space? For practical purposes the use of Eqs.5,6 for the transformation from position space into Bargmann Fock space is rather inconvenient. Let us therefore, starting from known expressions, develop some new and more direct formulas for the transformations from the Bargmann Fock basis to the position or momentum basis and vice versa.

Let a state $|\psi\rangle$ be given in the position basis by its wave function $\psi_{x}(x)=\langle x \mid \psi\rangle$. We can then calculate its Bargmann Fock function $|\psi\rangle=|\psi(\bar{\eta})\rangle$ as follows:

$$
\psi(\bar{\eta})=\left(2 \pi L^{2}\right)^{-1 / 4} \int_{-\infty}^{+\infty} d x e^{-\frac{1}{2} \bar{\eta}^{2}+\bar{\eta} \frac{x}{L}-\frac{1}{4}\left(\frac{x}{L}\right)^{2}} \psi_{x}(x)
$$

This result3 was obtained in [44], see also [45] and references therein.

The inverse transformation is:

$$
\psi_{x}(x)=\left(8 \pi^{3} L^{2}\right)^{-1 / 4} \int_{-i \infty}^{+i \infty} d \bar{\eta} e^{\frac{1}{2} \bar{\eta}^{2}-\bar{\eta} \frac{x}{L}+\frac{1}{4}\left(\frac{x}{L}\right)^{2}} \psi(\bar{\eta})
$$

group defines a set of intertwining morphisms of quantum group modules, which forms itself a representation of the braid group.

${ }^{3}$ Recall that this means that $\psi(\bar{\eta})$, read as a function of the Bargmann Fock operator $\bar{\eta}$, applied on $|1\rangle$ yealds the state which has the position wave function $\psi_{x}(x)$. 
To see this, a short calculation shows, that the Bargmann Fock function $\psi(\bar{\eta}):=1$ is mapped onto the position wave function

$$
\psi_{x}(x)=\left(2 \pi L^{2}\right)^{-1 / 4} e^{-1 / 4(x / L)^{2}}
$$

and vice versa. The induction is then completed by showing that multiplying the Bargmann Fock function with $\bar{\eta}$ just amounts to the action of $\left(x / L-2 L \partial_{x}\right) / 2$ on the wave function in position space.

These formulas, connecting the position space with the 'Bargmann Fock space', are the analogue of the fourier transformation formulas connecting the position space with the momentum space. Analogously one derives similar formulas that connect the Bargmann Fock space directly to momentum space:

$$
\psi(\bar{\eta})=\left(\frac{2 L^{2}}{\pi \hbar^{2}}\right)^{1 / 4} \int_{-\infty}^{+\infty} d p e^{\frac{1}{2} \bar{\eta}^{2}+2 i \bar{\eta} \frac{L p}{\hbar}-\left(\frac{L p}{\hbar}\right)^{2}} \psi_{p}(p)
$$

with the inverse:

$$
\psi_{p}(p)=\left(\frac{L^{2}}{2 \pi^{3} \hbar^{2}}\right)^{1 / 4} \int_{-\infty}^{+\infty} d \bar{\eta} e^{-\frac{1}{2} \bar{\eta}^{2}-2 i \bar{\eta} \frac{L p}{\hbar}+\left(\frac{L p}{\hbar}\right)^{2}} \psi(\bar{\eta})
$$

For the proof, note that $\langle p \mid 1\rangle=\left(\frac{2 L^{2}}{\pi \hbar^{2}}\right)^{1 / 4} e^{-\left(\frac{L p}{\hbar}\right)^{2}}$.

Let us remark that from Eqs.11,12 immediately follows that the transformation

$$
\tilde{f}(y):=\int_{-\infty}^{+\infty} d x e^{-\frac{1}{L^{2}}(x-y)^{2}} f(x)
$$

which yields a 'gaussian-diluted' function has actually an inverse:

$$
f(x)=\frac{1}{\pi L^{2}} \int_{-\infty}^{+\infty} d y e^{+\frac{1}{L^{2}}(x-i y)^{2}} \tilde{f}(i y)
$$

Using Eq.11 we calculate the Bargmann Fock function $\psi_{\left(x_{0}\right)}(\bar{\eta})$ of e.g the $\mathbf{x}$-eigenvector with eigenvalue $x_{0}$, i.e. of the $\delta$ - function at $x_{0}$ on position space:

$$
\psi_{\left(x_{0}\right)}(\bar{\eta})=\left(2 \pi L^{2}\right)^{-1 / 4} e^{-\frac{\bar{\eta}^{2}}{2}+\bar{\eta} \frac{x_{0}}{L}-\frac{1}{4}\left(\frac{x_{0}}{L}\right)^{2}}
$$

Now, putting this function into Eq.7 as one of its two arguments, we calculate the scalar product of a state, with a $\delta$-function in position space. This yields another formula for the transformation from Bargmann Fock to position space:

$$
\psi_{x}(x)=\frac{\left(2 \pi L^{2}\right)^{-1 / 4}}{2 \pi i} \int d \eta d \bar{\eta} e^{-\bar{\eta} \eta-\frac{\eta^{2}}{2}+\eta \frac{x}{L}-\frac{1}{4}\left(\frac{x}{L}\right)^{2}} \psi(\bar{\eta})
$$


In Bargmann Fock space the scalar product of states can actually also be expressed purely algebraically, namely in terms of derivatives [9]:

$$
\langle\psi \mid \phi\rangle=\left.\overline{\psi(\bar{\eta})} e^{\partial_{\eta} \partial_{\bar{\eta}}} \phi(\bar{\eta})\right|_{\eta=0=\bar{\eta}}
$$

Here the exponential is defined through its power series i.e. $e^{\partial_{\eta} \partial_{\bar{\eta}}}=\sum_{r=0}^{\infty} \frac{\partial_{\eta} \partial_{\bar{\eta}}}{r !}$. where the derivatives $\partial_{\eta}$ act from the right. The evaluation procedure is to carry out the differentiations and then to set $\eta$ and $\bar{\eta}$ equal to zero. The remaining number is the value of the scalar product. This can be done algebraically by using the Leibniz rule $\partial_{\bar{\eta}} \bar{\eta}-\bar{\eta} \partial_{\bar{\eta}}=1$ and its complex conjugate $\eta \partial_{\eta}-\partial_{\eta} \eta=1$. Note that we are using the left derivative $\partial_{\bar{\eta}}$ and the right derivative $\partial_{\eta}$, which means explicitly that e.g.

$$
\begin{aligned}
\partial_{\bar{\eta}} \bar{\eta}^{2} & =\partial_{\bar{\eta}} \bar{\eta} \bar{\eta}=\left(\bar{\eta} \partial_{\bar{\eta}}+1\right) \bar{\eta}=\bar{\eta} \partial_{\bar{\eta}} \bar{\eta}+\bar{\eta} \\
& =\bar{\eta}\left(\bar{\eta} \partial_{\bar{\eta}}+1\right)+\bar{\eta}=\bar{\eta} \bar{\eta} \partial_{\bar{\eta}}+\bar{\eta}+\bar{\eta}=2 \bar{\eta}
\end{aligned}
$$

but

$$
\eta^{2} \partial_{\eta}=\eta\left(\partial_{\eta} \eta+1\right)=\ldots=2 \eta
$$

Thus e.g.:

$$
\begin{aligned}
\left\langle\bar{\eta}^{2} \mid 2+3 \bar{\eta}^{2}\right\rangle & =\left.\eta^{2} e^{\partial_{\eta} \partial_{\bar{\eta}}}\left(2+3 \bar{\eta}^{2}\right)\right|_{\eta=0=\bar{\eta}} \\
& =\left.\eta^{2} \sum_{r=0}^{\infty} \frac{\partial_{\eta} \partial_{\bar{\eta}}}{r !}\left(2+3 \bar{\eta}^{2}\right)\right|_{\eta=0=\bar{\eta}} \\
& =\left.3 \eta^{2} \frac{\partial_{\eta}{ }^{2} \partial_{\bar{\eta}}{ }^{2}}{2} \bar{\eta}^{2}\right|_{\eta=0=\bar{\eta}}=6
\end{aligned}
$$

Since the scalar product formula Eq.7 relies on ordinary commutative integration over the complex plane, it cannot be used in the noncommutative case. We will however be able to use a generalisation of Eq.19 th on the noncommutative Bargmann Fock space [9, 10].

We put the Bargmann Fock functions of position eigenvectors Eq.17 into Eq.19 and obtain now a new formula for the transformation from Bargmann Fock to position space:

$$
\psi_{x}(x)=\left(2 \pi L^{2}\right)^{-1 / 4}\left(e^{-\frac{\eta^{2}}{2}+\eta \frac{x}{L}-\frac{1}{4}\left(\frac{x}{L}\right)^{2}} e^{\partial_{\eta} \partial_{\bar{\eta}}} \psi(\bar{\eta})\right)_{\eta=0=\bar{\eta}}
$$

and thus:

$$
\psi_{x}(x)=\left(2 \pi L^{2}\right)^{-1 / 4}\left(e^{-\frac{1}{2} \partial_{\bar{\eta}}^{2}+\frac{x}{L} \partial_{\bar{\eta}}-\frac{1}{4}\left(\frac{x}{L}\right)^{2}} \psi(\bar{\eta})\right)_{\bar{\eta}=0}
$$

The new transformation formula has the advantage that it can be evaluated algebraically, i.e. by using the Leibniz rule to commute the differentiation symbols to the right and setting $\bar{\eta}=0$. Such techniques are generalisable to the case of noncommutative $\bar{\eta}$ 's. A similar calculation is possible for the transformation from Bargmann Fock to momentum space.

\footnotetext{
${ }^{4}$ It can be used even in the fermionic case where one would usually apply Berezin integration [9].
} 


\subsubsection{Pointwise multiplication}

When we will later abandon the position and momentum spaces, which were classical manifolds, we will still be able to obtain all informations about positions or momenta out of the matrix elements of the position and momentum operators. However, the operators $x$ and $p$ will not have eigenvectors so that there will be nothing that could be called points in position or momentum space. However, we will work with quantum field theories in the path integral framework and fields have to be multiplied pointwise in position space in order to describe local interactions. We thus have to translate the notion of pointwise multiplication in position space, into Bargmann Fock space, before we can generalise it. To this end we use the formulas of the preceding subsection, which connect the position space with the Bargmann Fock space:

The pointwise multiplication $*$ of functions on position space translates into momentum space as the well known convolution product:

$$
\begin{aligned}
& (\psi * \phi)(x)=\psi_{x}(x) \phi_{x}(x) \\
& (\psi * \phi)(p)=(2 \pi \hbar)^{-1 / 2} \int_{-\infty}^{+\infty} d k \psi_{p}(k) \phi_{p}(p-k)
\end{aligned}
$$

To check this one takes two arbitrary functions on momentum space, transforms them into position space, multiplies them pointwise and transforms the resulting function back into momentum space. The result is the convolution product formula Eq.23.

In order to derive the formula for pointwise multiplication in Bargmann Fock space, we proceed the same way: We transform two arbitrary Bargmann Fock functions into position space using our new formula Eq.21, multiply them pointwise and transform the result back into Bargmann Fock space, using Eq.11:

$$
\begin{aligned}
(\psi * \phi)(\bar{\eta}) & =\left.\left(2 \pi L^{2}\right)^{-\frac{3}{4}} \int_{-\infty}^{+\infty} d x e^{-\frac{1}{2}\left(\bar{\eta}^{2}+\partial_{\bar{\eta}^{\prime}}^{2}+\partial_{\bar{\eta}^{\prime \prime}}^{2}\right)+\frac{x}{L}\left(\bar{\eta}+\partial_{\bar{\eta}^{\prime}}+\partial_{\bar{\eta}^{\prime \prime}}\right)-\frac{3}{4}(x / L)^{2}} \psi\left(\bar{\eta}^{\prime}\right) \phi\left(\bar{\eta}^{\prime \prime}\right)\right|_{0} \\
& =\left.\left(\frac{2}{3 \pi L^{2}}\right)^{\frac{1}{4}} e^{\frac{1}{3}\left(\left(\bar{\eta}+\partial_{\bar{\eta}^{\prime}}+\partial_{\bar{\eta}^{\prime \prime}}\right)^{2}-\frac{1}{2}\left(\bar{\eta}^{2}+\partial_{\bar{\eta}^{\prime}}^{2}+\partial_{\bar{\eta}^{\prime \prime}}^{2}\right)\right.} \psi\left(\bar{\eta}^{\prime}\right) \phi\left(\bar{\eta}^{\prime \prime}\right)\right|_{0}
\end{aligned}
$$

Here and in the following $\left.\right|_{0}$ will always stand for 'all differentiations evaluated at zero'.

Let us also mention a 'weighted' pointwise multiplication that we may call $*$ ':

$$
\left(\psi *^{\prime} \phi\right)(x):=\psi_{x}(x) \phi_{x}(x) e^{\frac{1}{4}\left(\frac{x}{L}\right)^{2}}
$$

In Bargmann Fock space this now takes a particularly simple form:

$$
\begin{aligned}
\left(\psi *^{\prime} \phi\right)(\bar{\eta}) & =\left.\left(2 \pi L^{2}\right)^{-\frac{3}{4}} \int_{-\infty}^{+\infty} d x e^{-\frac{1}{2}\left(\bar{\eta}^{2}+\partial_{\bar{\eta}^{\prime}}^{2}+\partial_{\bar{\eta}^{\prime \prime}}^{2}\right)+\frac{x}{L}\left(\bar{\eta}+\partial_{\bar{\eta}^{\prime}}+\partial_{\bar{\eta}^{\prime \prime}}\right)-\frac{1}{2}(x / L)^{2}} \psi\left(\bar{\eta}^{\prime}\right) \phi\left(\bar{\eta}^{\prime \prime}\right)\right|_{0} \\
& =\left.\left(2 \pi L^{2}\right)^{-\frac{1}{4}} e^{\bar{\eta} \partial_{\bar{\eta}^{\prime \prime}}+\left(\bar{\eta}+\partial_{\bar{\eta}^{\prime \prime}}\right) \partial_{\bar{\eta}^{\prime}}} \psi\left(\bar{\eta}^{\prime}\right) \phi\left(\bar{\eta}^{\prime \prime}\right)\right|_{0} \\
& =\left.\left(2 \pi L^{2}\right)^{-\frac{1}{4}} \psi\left(\bar{\eta}+\partial_{\bar{\eta}^{\prime}}\right) \phi\left(\bar{\eta}+\bar{\eta}^{\prime}\right)\right|_{0}
\end{aligned}
$$


Recall that the Bargmann Fock polynomials $\bar{\eta}^{m}$ read in the position eigenbasis (Eq.5):

$$
\left\langle x \mid \bar{\eta}^{m}\right\rangle=\sqrt{m !} H_{m}(x) e^{-\frac{1}{4}\left(\frac{x}{L}\right)^{2}}
$$

where $H_{m}$ is the $m$ 'th Hermite polynomial. The pointwise multiplication $*$ of these basis functions thus yields a function of even more rapid decay at infinity. Their

product is decreasing like $e^{-\frac{1}{2}\left(\frac{x}{L}\right)^{2}}$. On the other hand we see that the weighted multiplication $*^{\prime}$ would cancel one of the Gaußian factors and thus keep the asymptotic behaviour unchanged under the multiplication.

For field theory we will later discuss the possibility to use, as a technical trick, the simpler to handle $*^{\prime}$ instead of $*$. Choosing $L$ large (recall that the choice of $L$ is arbitrary and does not affect the theory), we approximate $*$, except only for its infrared behaviour.

\subsection{Generalised quantum mechanics in the Bargmann Fock basis}

In the following four subsections we deal with generalisations of quantum mechanics, which have been developed in [9, 10, 13]. The Bargmann Fock functions now become noncommutative. However they still span a Hilbert space of states and still operators can be represented as integral kernels. On each such Hilbert space we will represent a class of generalised Heisenberg algebras of positions and momenta. We focus on two typical examples and demonstrate how nonzero minimal uncertainties in positions and momenta arise from the generalised commutation relations. The study of possible generalisations of the notion of pointwise multiplication, which will later be needed for the description of local interactions, is new.

\subsubsection{Noncommutative Bargmann Fock spaces}

In section 2.1 we recalled that the Heisenberg algebra of the $\mathbf{x}$ and $\mathbf{p}$ can be represented on functions on position space $\langle x \mid \psi\rangle$, on functions on momentum space $\langle p \mid \psi\rangle$, or e.g. on functions $\psi(\bar{\eta})$ on Bargmann Fock space. The corresponding changes of the bases, i.e. the unitary transformations that map functions on Bargmann Fock space to functions on position or momentum space, and vice versa, were given.

Obviously, a generalised Heisenberg algebra that implies nonzero minimal uncertainties in positions and momenta, cannot act on functions in a position or momentum eigenbasis, like $\langle x \mid \psi\rangle$ or $\langle p \mid \psi\rangle$. This is simply because there will be no position or momentum eigenstates $|x\rangle,|p\rangle$ in the representations of the Heisenberg algebra. However, we will see that such Heisenberg algebras can still be represented on Hilbert spaces of now noncommutative Bargmann Fock functions $\psi(\bar{\eta})$.

Our proceeding is as follows: We start by giving two examples of noncommutative Bargmann Fock spaces. We discuss their properties and show e.g. that operators on 
these spaces can still be written also as integral kernels. Secondly then we find two generalised Heisenberg algebras that can be represented on these spaces. It is shown that these two Heisenberg algebras indeed imply the existence of nonzero minimal uncertainties in positions and momenta. Thus, as we said, there is then no position or momentum eigenbasis to which we could transform. We will discuss how all information on positions and momenta can still be obtained, namely from the matrix elements of the $\mathbf{x}_{i}$ and $\mathbf{p}_{j}$.

Explicitly, we consider e.g. the following noncommutative generalisation of $n$ - dimensional Bargmann Fock space: The states $|\psi\rangle$ are given as polynomials or power series $\psi(\bar{\eta})$ where the coordinate functions $\bar{\eta}_{i}$ and $\eta_{i}$ are now noncommutative and defined to obey $(q \geq 1)$, see $[9]$ :

$$
\begin{array}{rlrl}
\bar{\eta}_{a} \bar{\eta}_{b}-q \bar{\eta}_{b} \bar{\eta}_{a}=0 & \text { for } & a>b \\
\eta_{a} \eta_{b}-q \eta_{b} \eta_{a}=0 & \text { for } & a<b \\
\eta_{a} \bar{\eta}_{b}-q \bar{\eta}_{b} \eta_{a}=0 & \text { for } & a \neq b \\
\eta_{a} \bar{\eta}_{a}-\bar{\eta}_{a} \eta_{a}=\left(q^{2}-1\right) \sum_{i \leq a} \bar{\eta}_{i} \eta_{i}
\end{array}
$$

The scalar product of two Bargmann Fock polynomials or power series $\psi(\bar{\eta})$ and $\phi(\bar{\eta})$, later to be interpreted as states $|\psi\rangle,|\phi\rangle$, is unique. It can be calculated by a generalised formula Eq.19:

$$
\langle\psi \mid \phi\rangle=\left.\overline{\psi(\bar{\eta})} e_{1 / q}^{\partial_{\eta_{i}} \partial_{\bar{\eta}}} \phi(\bar{\eta})\right|_{\eta=0=\bar{\eta}}
$$

Here, the $q$ - exponential is defined as (each $i$ summed over)

$$
e_{1 / q}^{\partial_{\eta_{i}} \partial_{\bar{\eta}_{i}}}=\sum_{r=0}^{\infty} \frac{\left(\partial_{\eta_{i}} \partial_{\bar{\eta}_{i}}\right)^{r}}{[r]_{1 / q} !}
$$

where

$$
[r]_{1 / q}=1+q^{-2}+q^{-4}+\ldots+q^{-2(r-1)}=\frac{q^{-2 r}-1}{q^{-2}-1}
$$

and

$$
[r]_{1 / q} !=1 \cdot[2]_{1 / q} \cdot[3]_{1 / q} \cdot \ldots \cdot[r]_{1 / q}
$$

The evaluation of the scalar product formula Eq.32 is algebraic: The Leibniz rules, or commutation relations, are used to commute the $\partial_{\bar{\eta}}$ 's to the right and the $\partial_{\eta}$ 's to the left. One evaluates at $\bar{\eta}=0=\eta$, i.e. all terms except the constants are set zero. The remaining number is the value of the scalar product. The Leibniz rules and other relations can be written in a compact form: (the $i, j$ summed over)

$$
\partial_{\bar{\eta}^{a}} \bar{\eta}_{b}-q R_{i b}^{a j} \bar{\eta}_{j} \partial_{\bar{\eta}^{i}}=\delta_{a b} \quad \partial_{\bar{\eta}^{a}} \partial_{\bar{\eta}^{b}}-\frac{1}{q} R_{a b}^{i j} \partial_{\bar{\eta}^{j}} \partial_{\bar{\eta}^{i}}=0
$$




$$
\begin{array}{cc}
\partial_{\bar{\eta}^{a}} \partial_{\eta^{b}}-\frac{1}{q}\left(R^{-1}\right)_{b i}^{j a} \partial_{\eta^{j}} \partial_{\bar{\eta}} i=0 & \partial_{\eta^{a}} \partial_{\eta^{b}}-\frac{1}{q} R_{b a}^{j i} \partial_{\eta^{j}} \partial_{\eta^{i}}=0 \\
\eta_{a} \partial_{\eta^{b}}-q R_{j a}^{b i} \partial_{\eta^{i}} \eta_{j}=\delta_{a b} & \partial_{\bar{\eta}^{a}} \eta_{b}-q R_{a b}^{i j} \eta_{j} \partial_{\bar{\eta}^{i}}=0 \\
\bar{\eta}_{a} \partial_{\eta^{b}}-q R_{b a}^{j i} \partial_{\eta^{j}} \bar{\eta}_{i}=0 & 0
\end{array}
$$

where: (the $e_{i}^{j}$ are matrix units)

$$
R=q \sum_{i} e_{i}^{i} \otimes e_{i}^{i}+\sum_{i \neq j} e_{i}^{i} \otimes e_{j}^{j}+(q-1 / q) \sum_{i>j} e_{j}^{i} \otimes e_{i}^{j}
$$

This generalised Bargmann Fock formalism introduces only one parameter $q$. The formalism was derived in [9] where it was shown that it is a minimal generalisation under certain consistency conditions like the invariance of $*$ - structure, Poincaré series and positivity of the norm, see also [12]. The algebra of the Bargmann Fock generators is conserved under the action of the quantum group $S U_{q}(n)$ and invariant under the conjugation operation. Technically the $\bar{\eta}, \eta, \partial_{\bar{\eta}}, \bar{\eta}$ generate a Fun $S U_{q}(n)$-comodule algebra, which means that the linear action of the $S U_{q}(n)$ respects the commutation relations as well as the $*$ - structure. Thus the quantum group consists of linear quantum canonical transformations of the algebra.

Let us consider the above commutation relations of the Bargmann Fock generators in the one dimensional case, where they reduce to:

$$
\begin{array}{lrl}
\partial_{\bar{\eta}} \bar{\eta}-q^{2} \bar{\eta} \partial_{\bar{\eta}}=1 & \eta \partial_{\eta}-q^{2} \partial_{\eta} \eta & =1 \\
\bar{\eta} \partial_{\eta}-q^{2} \partial_{\eta} \bar{\eta}=0 & \partial_{\bar{\eta}} \eta-q^{2} \eta \partial_{\bar{\eta}}=0 \\
\eta \bar{\eta}-q^{2} \bar{\eta} \eta=0 & \partial_{\eta} \partial_{\bar{\eta}}-q^{2} \partial_{\bar{\eta}} \partial_{\eta}=0
\end{array}
$$

Using $n$ commuting copies of it we arrive at a second $n$ - dimensional generalisation of the Bargmann Fock construction (all $q_{i} \geq 1$ ):

$$
\begin{array}{lrl}
\partial_{\bar{\eta}_{i}} \bar{\eta}_{i}-q_{i}^{2} \bar{\eta}_{i} \partial_{\bar{\eta}_{i}}=1 & \eta_{i} \partial_{\eta_{i}}-q_{i}^{2} \partial_{\eta_{i}} \eta_{i}=1 \\
\bar{\eta}_{i} \partial_{\eta_{i}}-q_{i}^{2} \partial_{\eta_{i}} \bar{\eta}_{i}=0 & \partial_{\bar{\eta}_{i}} \eta_{i}-q^{2} \eta_{i} \partial_{\bar{\eta}_{i}}=0 \\
\eta_{i} \bar{\eta}_{i}-q_{i}^{2} \bar{\eta}_{i} \eta_{i}=0 & \partial_{\eta_{i}} \partial_{\bar{\eta}_{i}}-q_{i}^{2} \partial_{\bar{\eta}_{i}} \partial_{\eta_{i}}=0
\end{array}
$$

where Bargmann Fock generators of different indices commute. It provides a simple example of a Bargmann Fock construction where there is no quantum group symmetry of the commutation relations. This generalised Bargmann Fock space is parametrised by $n$ parameters $q_{i}$.

Our two examples are certainly not the most general possible consistent generalisations of the construction of Bargmann Fock Hilbert spaces. Let us however postpone

\footnotetext{
${ }^{5}$ Note that ${ }^{-}$is an anti algebra morphism, so that e.g. $\overline{\partial_{\bar{\eta}^{i}} \bar{\eta}_{j}}=\eta_{j} \partial_{\eta^{i}}$ (we defined the $\partial_{\eta}$ 's as right derivatives)
} 
the study of a more general case. We will see that the two Bargmann Fock spaces given above can already serve as representation spaces for interesting generalised Heisenberg algebras.

In both cases the Hilbert space is spanned, as usual, by the ordered polynomials $\bar{\eta}_{1}^{r_{1}} \bar{\eta}_{2}^{r_{2}} \cdot \ldots \cdot \bar{\eta}_{n}^{r_{n}}$ which are found to be still orthogonal and do now have the norm:

$$
\left\langle\bar{\eta}_{1}^{r_{1}} \bar{\eta}_{2}^{r_{2}} \cdot \ldots \cdot \bar{\eta}_{n}^{r_{n}} \mid \bar{\eta}_{1}^{r_{1}} \bar{\eta}_{2}^{r_{2}} \cdot \ldots \cdot \bar{\eta}_{n}^{r_{n}}\right\rangle=\prod_{i=1}^{n}\left[r_{i}\right]_{q} !
$$

in the first case and for the second kind of generalised Bargmann Fock space it is:

$$
\left\langle\bar{\eta}_{1}^{r_{1}} \bar{\eta}_{2}^{r_{2}} \cdot \ldots \cdot \bar{\eta}_{n}^{r_{n}} \mid \bar{\eta}_{1}^{r_{1}} \bar{\eta}_{2}^{r_{2}} \cdot \ldots \cdot \bar{\eta}_{n}^{r_{n}}\right\rangle=\prod_{i=1}^{n}\left[r_{i}\right]_{q_{i}} !
$$

One also proves that the Bargmann Fock operators $\partial_{\bar{\eta}^{i}}$ and $\bar{\eta}_{j}$ that act on the functions $\psi(\bar{\eta})$ as differentiation and multiplication operators, are still adjoint to each other in respect to the scalar product, i.e.:

$$
\left\langle\bar{\eta}_{i} \psi(\bar{\eta}) \mid \phi(\bar{\eta})\right\rangle=\left\langle\psi(\bar{\eta}) \mid \partial_{\bar{\eta}^{i}} \phi(\bar{\eta})\right\rangle \quad \text { and } \quad\left\langle\partial_{\bar{\eta}^{i}} \psi(\bar{\eta}) \mid \phi(\bar{\eta})\right\rangle=\left\langle\psi(\bar{\eta}) \mid \bar{\eta}_{i} \phi(\bar{\eta})\right\rangle
$$

This insures that position and momentum operators, defined e.g. (like in ordinary quantum mechanics) through linear combinations $x_{j} \propto\left(\bar{\eta}_{j}+\partial_{\bar{\eta}^{j}}\right)$ and $p_{j} \propto i\left(\bar{\eta}_{j}-\partial_{\bar{\eta}^{j}}\right)$ are still symmetric operators on our space of polynomials in $\bar{\eta}$ 's.

Before we study the position and momentum operators in more detail, let us mention how, just like in ordinary quantum mechanics, operators can be represented as integral kernels.

\subsubsection{Integral kernels}

It is still possible to represent operators $P$ on Bargmann Fock functions, expressed in terms of multiplication and differentiation operators $\bar{\eta}_{j}$ and $\partial_{\bar{\eta}_{j}}$, as integral kernels. Once the operator $P\left(\bar{\eta}, \partial_{\bar{\eta}}\right)$ is normal ordered, there is a simple rule how to get its integral kernel $G_{P}$, which is a function of $\bar{\eta}^{\prime}$ and $\eta$. Integrating any Bargmann Fock function $\psi(\bar{\eta})$ over $G_{P}\left(\bar{\eta}^{\prime}, \eta\right)$ leads then to a function of $\bar{\eta}^{\prime}$, which is $P \psi\left(\bar{\eta}^{\prime}\right)$ :

$$
\int d \bar{\eta} d \eta G_{P}\left(\bar{\eta}^{\prime}, \eta\right) e_{1 / q}^{\partial_{\eta_{i}} \partial_{\bar{\eta}_{i}}} \psi(\bar{\eta})=P \psi\left(\bar{\eta}^{\prime}\right)
$$

Here, the integration is meant to be our algebraic scalar product, which expresses the integration in terms of derivatives, i.e. we defined for notational convenience:

$$
\int d \bar{\eta} d \eta \overline{\psi(\bar{\eta})} e_{1 / q}^{\partial_{\eta_{i}} \partial_{\bar{\eta}_{i}}} \phi(\bar{\eta}):=\left.\overline{\psi(\bar{\eta})} e_{1 / q}^{\partial_{\eta_{i}} \partial_{\bar{\eta}_{i}}} \phi(\bar{\eta})\right|_{\eta=0=\bar{\eta}}
$$

\footnotetext{
${ }^{6}$ We hope it is not too confusing if we denote the 'multiplication by $\bar{\eta}_{j}$ ' operator with the same symbol as the 'coordinate function' $\bar{\eta}_{j}$. They do of course obey the same commutation relations.
} 
For this to work, the appropriate commutation relations between two copies (e.g. primed and unprimed) of the function space had to be calculated, see [10.

If we define e.g. the position operator as $x_{i}:=L_{i}\left(\bar{\eta}_{i}+\partial_{\bar{\eta}^{i}}\right)$, it has the integral kernel

$$
G_{x}\left(\bar{\eta}^{\prime}, \eta\right)=L\left(\bar{\eta}_{i}^{\prime} e_{1 / q}^{\bar{\eta}_{j}^{\prime} \eta_{j}}+e_{1 / q}^{\bar{\eta}_{j}^{\prime} \eta_{j}} \eta_{i}\right)
$$

and another example is the $n$ - dimensional isotropic harmonic oscillator $H:=\omega \bar{\eta}_{i} \partial_{\bar{\eta}^{i}}$ (summed over $i$ ). Since $H$ is hermitean, the time evolution operator $U=e^{-i\left(t_{f}-t_{i}\right) H}$ is unitary. The eigenvalues of $H$ are:

$$
H\left|\bar{\eta}_{1}^{r_{1}} \bar{\eta}_{2}^{r_{2}} \cdot \ldots \cdot \bar{\eta}_{n}^{r_{n}}\right\rangle=\omega\left[r_{1}+r_{2}+\ldots+r_{n}\right]_{q}\left|\bar{\eta}_{1}^{r_{1}} \bar{\eta}_{2}^{r_{2}} \cdot \ldots \cdot \bar{\eta}_{n}^{r_{n}}\right\rangle
$$

where $[r]_{q}$ is the ' $q$ - number', i.e. the partial geometric series:

$$
[r]_{q}:=1+q^{2}+q^{4}+\ldots+q^{2(r-1)}=\frac{q^{2 r}-1}{q^{2}-1}
$$

The integral kernel of $U$, i.e. the Greens function is then found to be [10]:

$$
G_{U}=\sum_{r=0}^{\infty} \frac{\left(\bar{\eta}_{i}^{\prime} \eta_{i}\right)^{r}}{[r]_{1 / q} !} e^{-i \omega\left(t_{f}-t_{i}\right)[r]}
$$

These are the results for our first kind of generalised Bargmann Fock construction, using the relations Eqs.28-38. The corresponding formulas for our second example of a noncommutative Bargmann Fock space (Eqs.42 44) are easily derived from the

one-dimensional case above. An important difference is that the eigenvalues of the operator $H:=\omega \bar{\eta}_{i} \partial_{\bar{\eta}^{i}}$ then read:

$$
H\left|\bar{\eta}_{1}^{r_{1}} \bar{\eta}_{2}^{r_{2}} \cdot \ldots \cdot \bar{\eta}_{n}^{r_{n}}\right\rangle=\omega\left(\left[r_{1}\right]_{q_{1}}+\left[r_{2}\right]_{q_{2}}+\ldots+\left[r_{n}\right]_{q_{n}}\right)\left|\bar{\eta}_{1}^{r_{1}} \bar{\eta}_{2}^{r_{2}} \cdot \ldots \cdot \bar{\eta}_{n}^{r_{n}}\right\rangle
$$

\subsubsection{Positions and momenta}

We now study the Heisenberg algebras of positions and momenta that can be represented on our generalised Bargmann Fock spaces. Let us start with the Heisenberg algebra that is generated by operators $\mathbf{x}_{r}, \mathbf{p}_{r},(r=1, \ldots, n)$ which are represented, just like in usual quantum mechanics, as

$$
\mathbf{x}_{r}=L_{r}\left(\bar{\eta}_{r}+\partial_{\bar{\eta}^{r}}\right) \quad \text { and } \quad \mathbf{p}_{r}=i K_{r}\left(\bar{\eta}_{r}-\partial_{\bar{\eta}^{r}}\right)
$$

on our first Bargmann Fock space. Note that since the $\partial_{\bar{\eta}^{i}}$ and the $\bar{\eta}_{j}$ do not carry units, the newly introduced constants $L_{r}$ and $K_{r}$ do.

The commutation relations of this Heisenberg algebra then read as follows:

$$
\left[\mathbf{x}_{r}, \mathbf{p}_{r}\right]=i \hbar+i \hbar\left(q^{2}-1\right) \sum_{s \leq r}\left(\frac{q^{2}+1}{2}\right)^{s-1}\left(\frac{\mathbf{x}_{s}^{2}}{4 L_{s}^{2}}+\frac{\mathbf{p}_{s}^{2}}{4 K_{s}^{2}}\right)
$$


The mixed commutation relations read for $s>r$ :

$$
\left[\mathbf{x}_{s}, \mathbf{p}_{r}\right]=-i \frac{K_{r}}{L_{r}} \frac{q-1}{q+1}\left\{\mathbf{x}_{s}, \mathbf{x}_{r}\right\} \quad\left[\mathbf{x}_{s}, \mathbf{x}_{r}\right]=-i \frac{L_{r}}{K_{r}} \frac{q-1}{q+1}\left\{\mathbf{x}_{s}, \mathbf{p}_{r}\right\}
$$

For $s<r$ one gets:

$$
\left[\mathbf{x}_{s}, \mathbf{p}_{r}\right]=i \frac{L_{s}}{K_{s}} \frac{q-1}{q+1}\left\{\mathbf{p}_{s}, \mathbf{p}_{r}\right\} \quad\left[\mathbf{p}_{s}, \mathbf{p}_{r}\right]=-i \frac{K_{s}}{L_{s}} \frac{q-1}{q+1}\left\{\mathbf{x}_{s}, \mathbf{p}_{r}\right\}
$$

In ordinary quantum mechanics where $[\mathbf{x}, \mathbf{p}]=i \hbar$ the $L_{i}$ and $K_{j}$ must obey $L_{r} K_{r}=$ $\hbar / 2$, in order to get exactly the term $i \hbar$ on the lhs. In our generalised situation, in order to get in the commutation relations Eq.56 also exactly the term $i \hbar$, the $L_{r}$ and $K_{r}$ must obey

$$
L_{r} K_{r}:=\frac{\hbar}{2}\left(\frac{q^{2}+1}{2}\right)^{r}
$$

That the constant term on the rhs is exactly $i \hbar$ without any $q$-factors, insures that the generalised framework describes ordinary quantum mechanical behaviour in medium scales, see [13]. Recall that we want to develop generalisations that deviate from ordinary quantum theory only in the very small and large scales.

Let us also consider our second Bargmann Fock space. Using again the usual definitions Eq.55 we can represent on it the Heisenberg algebra with the commutation relations:

$$
\begin{gathered}
{\left[\mathbf{x}_{i}, \mathbf{p}_{j}\right]=i \hbar \delta_{i j}+i \hbar \delta_{i j}\left(q^{2}-1\right)\left(\frac{1}{4 L_{i}^{2}} \mathbf{x}_{i}^{2}+\frac{1}{4 K_{i}^{2}} \mathbf{p}_{i}^{2}\right)} \\
{\left[\mathbf{x}_{i}, \mathbf{x}_{j}\right]=0} \\
{\left[\mathbf{p}_{i}, \mathbf{p}_{j}\right]=0}
\end{gathered}
$$

where

$$
L_{i} K_{i}=\hbar\left(q^{2}+1\right) / 4
$$

Note that if $q=1$, or for the second Heisenberg algebra if all $q_{i}=1$, the constants $K_{i}$ and $L_{i}$ drop out of the commutation relations. This reflects that in ordinary quantum mechanics a length or a momentum scale can only be set by the Hamiltonian i.e. by choosing a particular system. Here, for $q>1$, or some $q_{i}>1$, the $K_{r}$ and $L_{s}$ do appear in the commutation relations. These scales now become a property of the quantum mechanical formalism itself. Thus, although the generalisation parameters $q_{i}$ of the Bargmann Fock spaces are unitless, there do now appear length and momentum scales in the framework.

As we said, one can show that our generalised Heisenberg algebras describe ordinary quantum mechanical behaviour in the physical region of not too large and not too small positions and momenta, explicitly where:

$$
\left(\Delta x_{0}\right)^{2} \ll\left\langle\mathbf{x}^{2}\right\rangle \ll \frac{\hbar^{2}}{4\left(\Delta p_{0}\right)^{2}} \quad \text { and } \quad\left(\Delta p_{0}\right)^{2} \ll\left\langle\mathbf{p}^{2}\right\rangle \ll \frac{\hbar^{2}}{4\left(\Delta x_{0}\right)^{2}}
$$


This is nicely consistent with the wave-particle dualism, because it means that the critical upper length scale, where the formalism begins to deviate significantly, is just the length needed to measure momentum to its minimal uncertainty. Similarly, the critical upper momentum is just the momentum needed to measure the position to its minimal uncertainty. Note that the existence of this region of effectively ordinary quantum theoretical behaviour relies on the fact that in our generalised Heisenberg algebras the constant term on the rhs is exactly $i \hbar \delta_{i j}$ without any $q$ - factors [13].

The functional analysis of our position and momentum operators is as follows [10]: We start by defining their domain $D$ to be the set of only those Bargmann Fock functions that are polynomials in $\bar{\eta}$ 's. This is a dense set in the Hilbert space and on it the $\mathbf{x}_{i}$ and $\mathbf{p}_{i}$ are symmetric with their ranges in the domain. The $\mathbf{x}_{i}$ and $\mathbf{p}_{i}$ are no longer essentially self-adjoint. Their adjoints $\mathbf{x}_{i}^{*}$ and $\mathbf{p}_{i}^{*}$ are closed but nonsymmetric. The $\mathbf{x}_{i}^{* *}$ and $\mathbf{p}_{i}^{* *}$ are closed and symmetric, but their deficiency indices no longer vanish. The deficiency subspaces are still of the same size so that there are continuous families of self adjoint extensions.

One might be tempted to try to fix the choice of the self adjoint extensions by the requirement that the domains coincide. A diagonalisation of the $\mathbf{x}_{i}$ or the $\mathbf{p}_{i}$ would then lead as usual to momentum space or position space representations. However, one can show that there are no more eigenstates in the representations of the Heisenberg algebras, and there is thus no position or momentum eigenbasis.

The mechanism is the same for both of our examples of generalised Heisenberg algebras. It can already be seen in the one-dimensional case that non of the observables $\mathbf{x}$ or $\mathbf{p}$ is diagonalisable on a representation of the Heisenberg algebra, nor does it have any eigenvectors:

The commutation relation

$$
[\mathbf{x}, \mathbf{p}]=i \hbar+i \hbar\left(q^{2}-1\right)\left(\frac{1}{4 L^{2}} \mathbf{x}^{2}+\frac{1}{4 K^{2}} \mathbf{p}^{2}\right)
$$

leads to the uncertainty relation:

$$
\Delta x \Delta p \geq \frac{\hbar}{2}\left(1+\left(q^{2}-1\right)\left(\frac{(\Delta x)^{2}+\langle\mathbf{x}\rangle^{2}}{4 L^{2}}+\frac{(\Delta p)^{2}+\langle\mathbf{p}\rangle^{2}}{4 K^{2}}\right)\right)
$$

It implies nonzero minimal uncertainties in $\mathbf{x}$ as well as $\mathbf{p}$ measurements. This can be seen as follows: As e.g. $\Delta x$ gets smaller, $\Delta p$ must increase so that the product $\Delta x \Delta p$ of the lhs remains larger than the rhs. In usual quantum mechanics this is always possible, i.e. $\Delta x$ can be made arbitrarily small. However, in the generalised case, where $q>1$, there is a $(\Delta p)^{2}$ term on the rhs which eventually grows faster with $\Delta p$ than the lhs. Thus $\Delta x$ can no longer become arbitrarily small. The minimal uncertainty in position measurements comes out as:

$$
\Delta x_{0}=L \sqrt{1-q^{-2}}
$$


Analogously one obtains the smallest uncertainty in the momentum:

$$
\Delta p_{0}=K \sqrt{1-q^{-2}}
$$

where we have from Eq.59 that $K=\left(q^{2}+1\right) \hbar / 4 L$. For the details on the functional analysis and the uncertainty relation see [13], also for the $n$ dimensional case which is analogous.

The essential point is, that due to the nonvanishing minimal uncertainties in positions and momenta, there can no longer be $\mathbf{x}$ or $\mathbf{p}$ eigenstates in the representations of the Heisenberg algebra. To see this, recall that if we had an $\mathbf{x}$ eigenstate $\left|x_{0}\right\rangle$ with $\mathbf{x} .\left|x_{0}\right\rangle=x_{0}\left|x_{0}\right\rangle$ it would have no uncertainty in the position measurement

$$
(\Delta x)_{\left|x_{0}\right\rangle}^{2}=\left\langle x_{0}\left|\left(\mathbf{x}-\left\langle x_{0}|\mathbf{x}| x_{0}\right\rangle\right)^{2}\right| x_{0}\right\rangle=0
$$

which would be a contradiction. There are thus no physical states which are eigenstates of $\mathbf{x}$ or $\mathbf{p}$. We should clarify how we identify the space of physical states, which is actually smaller than e.g. our Hilbert space of (weighted) square integrable Bargmann Fock functions: The space of physical states has of course to be a representation space on which the expectation values of positions and momenta are well defined. Mathematically this means that it has to be a representation of the Heisenberg algebra (i.e. not of $\mathbf{x}$ or of $\mathbf{p}$ alone!) on which both, $\mathbf{x}$ and $\mathbf{p}$ are symmetric and have their ranges in the domain. The space $D$ of Bargmann Fock polynomials is such a physical domain. However, its completion with respect to the ordinary norm is too large to be a representation of the Heisenberg algebra. It should be interesting to find a suitable norm with respect to which our physical domain $D$ could be completed to a domain $\bar{D}$, which would be a representation of the Heisenberg algebra, i.e. on which $\mathbf{x}$ and $\mathbf{p}$ would remain symmetric while also having their range in the domain.

We will see how the nontrivial functional analytic behaviour of $\mathbf{x}$ and $\mathbf{p}$, i.e. their non essentially-self-adjointness manifests itself also in the physical description of positions and momenta.

Recall that all information on positions and momenta is contained in the matrix elements of the position and momentum operators, and matrix elements can of course be calculated in any basis. In our Bargmann Fock basis matrix, elements of e.g. the position operators are calculated as

$$
\langle\psi|\mathbf{x}| \phi\rangle=\left.\overline{\psi(\bar{\eta})} e_{1 / q}^{\partial_{\eta} \partial_{\bar{\eta}}} L\left(\bar{\eta}+\partial_{\bar{\eta}}\right) \phi(\bar{\eta})\right|_{0}
$$

In particular, we can calculate Bargmann Fock functions that denote states $\psi_{\left(x^{\prime}\right)}(\bar{\eta})$, which approximate position eigenstates to the eigenvalue $x^{\prime}$. To this end we start with the eigenvalue equation:

$$
\mathbf{x} \cdot\left|\psi_{\left(x^{\prime}\right)}\right\rangle=x^{\prime}\left|\psi_{\left(x^{\prime}\right)}\right\rangle \quad \text { i.e. } \quad L\left(\bar{\eta}+\partial_{\bar{\eta}}\right) \psi_{\left(x^{\prime}\right)}(\bar{\eta})=x^{\prime} \psi_{\left(x^{\prime}\right)}(\bar{\eta})
$$


Expanding $\psi_{\left(x^{\prime}\right)}(\bar{\eta})$ in the $\bar{\eta}$ 's yields a recursion formula for the coefficients of the expansion. In ordinary quantum mechanics the solution is the Dirac $\delta$ 'function', transformed into Bargmann Fock space, i.e. Eq.17 (with $x^{\prime}$ instead of $x_{0}$ ). In the generalised setting the (infinite) recursion can also be solved, but now the power series that solves it is no longer contained in the representation of the Heisenberg algebra, since this would of course contradict the uncertainty relation. However, every polynomial approximation of the power series is contained in the above defined domain $D$. It is thus contained in the representation of the Heisenberg algebra, and is therefore a physical state. Using such states we can still describe very localised particles. We can even use approximating polynomials of arbitrarily high degree, and thus come 'nearer and nearer' to the above described power series that would be a position eigenvector. However, and this is now an important effect of the non essential self-adjointness of our $\mathbf{x}$ and $\mathbf{p}$ - operators, no polynomial describes a localisation that is more precise than $\Delta x_{0}$. Similarly, in this way we can also calculate states that describe particles of relatively precise momentum, however of course not more precise than $\Delta p_{0}$.

In contrast to the $\delta$ - 'functions' of ordinary quantum theory, these approximating states are no longer orthogonal to each other (not even the unphysical power series that would solve the eigenvalue equation are orthogonal). For more details and a graph of their scalar product see [13]. It should also be interesting to study whether a generalised fourier transformation can be found that would allow to easily transform information on positions into information on momenta. In this context, compare also with the generalised quantum mechanics (with discrete $x$ and $p$ spectra) developed in [28, 29], where techniques developed in [30] allow such generalised fourier transformations.

To summarise, we arrived at the following picture:

While in classical mechanics the states can have exact positions and momenta, in quantum mechanics there is the uncertainty relation that does not allow $\mathbf{x}$ and $\mathbf{p}$ to have common eigenvectors. Nevertheless $\mathbf{x}$ and $\mathbf{p}$ separately do have 'eigenvectors', though non-normalisable ones. The spectrum is continuous, namely the whole configuration or momentum space.

Our 'noncommutative geometry'- or quantum group generalisations of the Heisenberg algebra have further consequences for the observables $\mathbf{x}$ and $\mathbf{p}$ : It is not only that the $\mathbf{x}$ and $\mathbf{p}$ have no common eigenstates. The uncertainty relation now implies that they even have no eigenvectors at all in the representation of the Heisenberg algebra. This means of course the non-existence of absolute precision in position or momentum measurements. Instead there are nonzero minimal uncertainties in these measurements.

Let us discuss the level of generalisation that we have achieved so far.

The two generalised Heisenberg algebras (Eqs.56-58 and Eqs.60-63) that we have studied are special examples of Heisenberg algebras with commutation relations of 
the form (summed over repeated indices):

$$
\begin{gathered}
{\left[\mathbf{x}_{r}, \mathbf{p}_{s}\right]=i \hbar \delta_{r s}+i \hbar A_{r s, t u}\left\{\mathbf{x}_{t}, \mathbf{x}_{u}\right\}+i \hbar B_{r s, t u}\left\{\mathbf{p}_{t}, \mathbf{p}_{t}\right\}} \\
{\left[\mathbf{x}_{r}, \mathbf{x}_{s}\right]=i C_{r s, t u}\left\{\mathbf{x}_{t}, \mathbf{p}_{u}\right\}} \\
{\left[\mathbf{p}_{r}, \mathbf{p}_{s}\right]=i D_{r s, t u}\left\{\mathbf{x}_{t}, \mathbf{p}_{u}\right\}}
\end{gathered}
$$

where the $A, B, C, D$ are real matrices, i.e. all $A_{r s, t u} \in \mathbf{R}$ etc.

We now know that e.g. if the diagonals of the matrices $A$ and $B$ are positive, which is e.g. the case in our examples, this leads to the appearance of nonzero minimal uncertainties in momenta and in positions respectively.

We saw in particular how such a matrices $B$ can introduce a term proportional to $(\Delta p)^{2}$ into the uncertainty relations. As we will be discussing later in this paper, exactly this type of correction to the uncertainty relation has actually already been suggested to appear at small scales like the Planck scale.

On the other hand we saw how the introduction of a matrix $A$ leads to the appearance of nonzero minimal uncertainties in momentum. As we said, we expect that ordinary large scale gravity, i.e. curvature, causes such uncertainties in momentum. Therefore it has to be shown that the momentum operators, defined as the generators of translations, obey commutation relations like those obtained from the introduction of a matrix $A$, when working on a space which is flat only up to first order corrections. A forthcoming paper on this subject is in progress.

In the present paper we are however merely interested in quantum theory alone and its generalisability. We were naturally led to consider Heisenberg algebras of the form of Eqs.72-74. We dealt with two examples of Heisenberg algebras of this general form which we represented on our $q$ - Bargmann Fock spaces. These Heisenberg algebras have $n$ length scales $L_{i}$ and 1 dimensionless parameter $q$ or $n$ dimensionless $q_{i}$ 's respectively. However, let us note that more Heisenberg algebras of the form 7274 can be represented on our $q$ - Bargmann Fock spaces. This is because, whenever we have a representation of a Heisenberg algebra specified by matrices $A, B, C$, D, we can on the same space also represent an entire class of Heisenberg algebras. This class consists of the Heisenberg algebras specified by matrices $A^{\prime}, B^{\prime}, C^{\prime}, D^{\prime}$, where

$$
\begin{aligned}
A_{a b, c d}^{\prime} & =M_{a i}^{-1} M_{j b} M_{k c} M_{l d} A_{i j, k l} \\
B_{a b, c d}^{\prime} & =M_{a i}^{-1} M_{b j} M_{c k}^{-1} M_{d l}^{-1} B_{i j, k l} \\
C_{a b, c d}^{\prime} & =M_{a i}^{-1} M_{b j}^{-1} M_{k c} M_{d l}^{-1} C_{i j, k l} \\
D_{a b, c d}^{\prime} & =M_{i a} M_{j b} M_{c k}^{-1} M_{l d} D_{i j, k l}
\end{aligned}
$$

for arbitrary $M \in G L(n, \mathbf{R})$. To see this, note that all Heisenberg algebras within one class are isomorphic with the isomorphism given on the generators as

$$
\mathbf{x}_{r} \rightarrow \mathbf{x}_{r}^{\prime}=M_{r s}^{-1} \mathbf{x}_{s} \quad \mathbf{p}_{r} \rightarrow \mathbf{p}_{r}^{\prime}=M_{s r} \mathbf{p}_{s}
$$


Nevertheless, since the actual commutation relations between the position and momentum operators are not invariant, these Heisenberg algebras all describe different physical behaviour. Note in particular that the functional analysis of the $\mathbf{x}_{i}$ and $\mathbf{p}_{j}$ changes drastically when there are negative diagonal elements of the matrices $A$ and $B$, (see [13]). This case deserves further study.

To summarise, we can now represent two classes of generalised Heisenberg algebras on $q$ Bargmann Fock Hilbert spaces. They will serve us as examples for our studies of the influence of nonzero minimal $\mathbf{x}$ and $\mathbf{p}$ - uncertainties on the infrared and ultraviolet behaviour in quantum field theories. We postpone the explicit construction of the Hilbert space representations of generalised Heisenberg algebras that are characterised by fully arbitrary real matrices $A, B, C, D$. The appearance of nonzero minimal position and momentum uncertainties then follows from the uncertainty relations, e.g. in the case of positive diagonals of the matrices $A$ and $B$.

\subsubsection{Pointwise multiplication}

The notion of pointwise multiplication is used for the description of local interactions in quantum field theory. In our generalised framework there are no more position eigenstates, i.e. there are no more 'points in position space'. One must now develop a generalised notion of 'noncommutative locality'.

In ordinary quantum mechanics, states $|\psi\rangle$ can be expanded in the position eigenbasis $\{|x\rangle\}$ to obtain wave functions on position space $\psi_{x}(x)=\langle x \mid \psi\rangle$. Their pointwise multiplication

$$
(\psi * \phi)(x):=\psi_{x}(x) \phi_{x}(x)
$$

is the convolution product in momentum space and it reads in Bargmann Fock space for $n$ dimensions (see Eq.24):

$$
(\psi * \phi)(\bar{\eta})=\left.\left(\frac{2}{3 \pi L^{2}}\right)^{\frac{n}{4}} e^{\sum_{i=1}^{n} \frac{1}{3}\left(\left(\bar{\eta}_{i}+\partial_{\bar{\eta}_{i}^{\prime}}+\partial_{\bar{\eta}_{i}^{\prime \prime}}\right)^{2}-\frac{1}{2}\left(\bar{\eta}_{i}^{2}+\partial_{\bar{\eta}_{i}^{\prime}}^{2}+\partial_{\bar{\eta}_{i}^{\prime \prime}}^{2}\right)\right.} \psi\left(\bar{\eta}^{\prime}\right) \phi\left(\bar{\eta}^{\prime \prime}\right)\right|_{0}
$$

The weighted pointwise multiplication $\left(\psi *^{\prime} \phi\right)(x):=\psi_{x}(x) \phi_{x}(x) e^{\frac{x_{i} x_{i}}{4 L^{2}}}$ reads in $n$ dimensional Bargmann Fock space simply, see Eq.26:

$$
\left(\psi *^{\prime} \phi\right)(\bar{\eta})=\left.\left(2 \pi L^{2}\right)^{-\frac{n}{4}} \psi\left(\bar{\eta}+\partial_{\bar{\eta}^{\prime}}\right) \phi\left(\bar{\eta}+\bar{\eta}^{\prime}\right)\right|_{0}
$$

These formulas are obtained by taking two functions on Bargmann Fock space, transforming them into position space, multiplying them pointwise and then transforming the result back into Bargmann Fock space.

An analogous proceeding is of course not possible in our generalised quantum mechanics. The position operators $\mathbf{x}_{i}$ do no longer have eigenvectors in the representation of the Heisenberg algebra. We abandoned the position eigenbasis and thus also functions on position space which could be multiplied pointwise. We succeeded in abandoning the notion of points, but what should now be the generalised notion 
of 'noncommutative locality'? For the pointwise multiplication one may be tempted to simply stick to Eq.81, even for $q>1$. However, writing the exponential as a power series there are terms like e.g. $\bar{\eta}_{i}^{2} \bar{\eta}_{j}^{2}$ or equally $\bar{\eta}_{j}^{2} \bar{\eta}_{i}^{2}$. In the generalised framework such terms are no longer equal, due to the noncommutativity. There is thus no unique generalisation of Eq.81.

Clearly the noncommutative generalisation of the notion of pointwise multiplication, i.e. of locality, has to be developed on physical grounds. This is a very crucial point in our generalisation of quantum theory and we will here not try to give a general answer. Let us however note, that the weighted pointwise multiplication $*^{\prime}$ does appear to be straightforwardly generalisable since it does not involve any functions other than the two Bargmann Fock functions that are to be multiplied. In spite of its modified infrared behaviour we will later use $*^{\prime}$ as a working hypothesis.

\section{Quantum field theory with nonzero minimal un- certainties}

We will be working with the path integral approach to quantum field theory. Our aim is to generalise its Heisenberg algebra of 4- positions and 4- momenta. In quantum field theory one would usually focus on the Heisenberg algebra of the fields and their conjugate momentum fields. Let us therefore clarify the rôle of this Heisenberg algebra of the positions and the momenta, which we are going to generalise.

In quantum field theory too there is a Heisenberg algebra generated by the $\mathbf{x}_{\mu}$ and $\mathbf{p}_{\nu}$, which, in the euclidean case, obey the commutation relations $\left[\mathbf{x}_{\mu}, \mathbf{p}_{\nu}\right]=i \hbar \delta_{\mu \nu}$. This Heisenberg algebra is represented on the (Hilbert) space of fields $\phi$. The quantum field theoretical partition function $Z=\int D \phi e^{i S[\phi] / \hbar}$ is the sum over all amplitudes $e^{i S[\phi] / \hbar}$ where $S$ is the action functional and the e.g. bosonic fields $\phi$ run through the representation space of the Heisenberg algebra.

A 'field on position space' is given by the coordinates $\phi(x)$ of the abstract field $\phi$ in the position eigenbasis. In this basis the scalar product of fields reads $\left(\phi(x), \phi^{\prime}(x)\right)=$ $\int d^{4} x \phi(x) \phi^{\prime}(x)$. The scalar product of momentum and position eigenvectors $\phi_{p}$ and $\phi_{x}$, is $\left(\phi_{p}, \phi_{x}\right)=(2 \pi \hbar)^{-2} e^{-i x \cdot p / \hbar}$. Therefore the unitary transformation to the field's coordinates in the momentum eigenbasis reads: $\phi(p)=\int d^{4} x\left(\phi_{p}, \phi_{x}\right) \phi(x)$.

Analogous to the situation in quantum mechanics, the position and momentum operators are essentially self-adjoint with (non-normalisable) 'eigenvectors', namely plane waves or Dirac $\delta$ - peaks. These eigenvectors still] are the 'points' in position or in momentum space, which means that e.g. the coordinates $p_{\nu}$ of a 'point' in momentum space are eigenvalues of the operators $\mathbf{p}_{\nu}$.

\footnotetext{
${ }^{7}$ This is analogous to the situation in quantum mechanics. Positions and momenta do not become mere 'parameters' in quantum field theory.
} 
Choosing an arbitrary positive length $L$ we define Bargmann Fock operators $\bar{\eta}_{\mu}:=$ $\frac{1}{2 L} \mathbf{x}_{\mu}-\frac{i L}{\hbar} \mathbf{p}_{\mu}$ and $\partial_{\bar{\eta}_{\mu}}:=\frac{1}{2 L} \mathbf{x}_{\mu}+\frac{i L}{\hbar} \mathbf{p}_{\mu}$. Fields can then be written as Bargmann Fock functions $\phi=\phi(\bar{\eta})$. Recall that $\phi(\bar{\eta})$ denotes the field obtained by applying the function $\phi(\bar{\eta})$ of the Bargmann Fock operators $\bar{\eta}$ on that field which is eigenvector to all $\partial_{\bar{\eta}_{\mu}}$ with the eigenvalue 0 (a gaußian), see end of Sec.Đ.

Also, arbitrary fields $\phi$ can be expanded in the countable eigenbasis of the operator $\bar{\eta}_{\mu} \partial_{\bar{\eta}_{\mu}}$ to obtain the field's coordinates $\phi_{r_{1}, r_{2}, r_{3}, r_{4}}$ in this Bargmann Fock basis. Similarily, the operators on the fields can be expanded in the Bargmann Fock basis. The scalar product reads $\left(\phi, \phi^{\prime}\right)=\sum_{r_{1}, r_{2}, r_{3}, r_{4}}^{\infty} \phi_{r_{1}, r_{2}, r_{3}, r_{4}} \phi_{r_{1}, r_{2}, r_{3}, r_{4}}^{\prime}$ in this basis.

The path integral is usually written in the position eigenbasis, which means to work with with the field's coordinates $\phi(x)$ in the position eigenbasis. The functional $S$ is then also written in the position eigenbasis i.e. it is expressed in terms of integrals and operators on functions over position space. It is often more convenient, e.g. for the derivation of Feynman rules, to consider the path integral, i.e. the same abstract fields $\phi$ and the same abstract action functional $S$ in the momentum eigenbasis instead. The action functional $S$ is then expressed in terms of integrals and operators on functions over momentum space.

Similarly, and this will be our choice, we can consider the path integral, i.e. the same abstract fields $\phi$ and the same abstract action functional $S$ in the Bargmann Fock basis of the function space. Before we further discuss quantum field theory in the Bargmann Fock basis, let us come back to the (of course basis independent) meaning of the operators $\mathbf{x}_{\mu}, \mathbf{p}_{\nu}$ and the fields $\phi$ that they are acting on.

In relativistic quantum field theory, the rôle of the 4-position and 4-momentum operators, the fields and their propagators is not as simple as in quantum mechanics. The coordinates $\phi(x)$ of a field $\phi$ in the position eigenbasis are not the 'probability amplitude for finding a particle at $x$ ', and Greens functions $G_{2}\left(x_{2}, x_{1}\right)$ do not simply propagate particles from $x_{1}$ to $x_{2}$ : It is not sufficient to know the amplitudes on a given spacelike surface to be able to calculate the amplitude at some point $P$ in the future, even when neglecting interactions. Problems with Newton Wigner localisationand propagation are related to that, see e.g. [33. The reason for this is the unavoidable existence of antiparticles:

What has to be known in order to calculate the field's amplitude at a point $P$ in space-time is not only the amplitudes of positive frequencies on an earlier spacelike surface but also the amplitudes of negative frequencies on a later spacelike surface, since they both propagate towards $P$, see e.g. [50]. This is to be compared with the relativistic propagation of an ordinary measurable field, e.g. a classical electrical potential or a directly measurable probability wave. One would then apply retarded boundary conditions to the equation of motion. This would ensure causal propagation of the wave (with the propagator vanishing for spacelike distancies). The knowledge of the field on a spacelike surface would be sufficient to calculate its values at later 
times.

However, in quantum field theory, not the fields are measurable. It is the particles, or more precisely, it is the expectation values of their 4-positions and 4-momenta that are, in principles, measurable. Therefore the boundary conditions for the propagators must be such that the particles propagate causally, rather than the unobservable 'waves'. This makes a difference because while the positive energy components, i.e. the particles are of course to be propagated forward in time, i.e. retarded, the negative energy components, i.e. the anti-particles components are to be propagated backwards in time, i.e. with advanced boundary conditions.

As was pointed out by Feynman [51, it is not difficult to see that it is relativity which does not allow a restriction to positive frequency components alone: Let us transform the propagator of the positive frequencies only, into the time coordinate. A theorem in fourier theory then applies, which tells that the fourier transform of a function that is nonzero only on a half axis, cannot vanish on any finite interval.

Therefore, the propagation of positive frequencies cannot vanish for any finite time interval. In particular, positive frequencies are also propagated between events, say E1 and E2, that are spacelike separated. In a suitable chosen Lorentz transformed frame the events E1 and E2 occur in the reversed order. In this frame, negative frequencies propagated backwards in time. This explains why relativity requires antiparticles, and why the antiparticle's properties are determined by the properties of the particles. We saw on the other hand that since not all frequencies can be propagated forward in time, propagation also in spacelike distances is unavoidable. E.g. for free massive particles there is an exponentially decaying propagation outside the lightcone. One might be surprised about this result since from the operator approach to quantum field theory one knows that spacelike separated field operators commute and also, naivly interpreted, it seems to contradict causality.

Recall however that the fields that are propagated by the quantum field theoretical propagators are not directly observable. In particular, space-time and momentumenergy are not classical parameter spaces. The information on space, time, momentum and energy is encoded in the formal expectation values of the corresponding operators in the Heisenberg algebra. These expectation values obey the uncertainty relations that are implied by the commutation relations of the position and momentum operators $\mathbf{x}_{\mu}$ and $\mathbf{p}_{\nu}$.

In particular, there is a time-energy uncertainty relation. More precisely, the energyand the time coordinates are related by a unitary mapping, which is found to be a fourier transformation. Any restriction, i.e. any knowledge on the energy, automatically leads to consequences, or 'uncertainties' in $x_{0}$, i.e. in time. We saw an example

\footnotetext{
${ }^{8}$ When speaking of 'in principle possible' measurements we are here and in the following not distinguishing virtual and real particles. Let us omit the discussion that for actual measurements on real particles one would consider sufficiently well separated wave packets of in- or out-going particles, each on the mass shell, the S-matrix limit etc.
} 
of exactly this above, namely that propagation restricted fo positive frequencies implies nonzero propagation to all finite time intervals, and hence also to spacelike distances. The reason why e.g. the propagator for massive particles cannot vanish outside the lightcone (but has an exponential tail instead) is therefore the following:

In order to decide whether particles propagate outside the lightcone one measures not only their expectation values of position and time. One also measures whether a particle has arrived at all, i.e. one measures also whether positive energy has arrived. The simple knowledge that positive energy has arrived however implies, as we said, by fourier transformation that any finite time interval has a nonvanishing probability of being the time of measurement. In general, the more precisely one measures the arriving particle's energy the more uncertain becomes the time of measurement and thus, whether the time of measurement was in- or outside the light cone.

On the other hand, one may decide to measure instead the time of the particles arrival very precisely. However, any sharp localisation in time, like e.g. a gaußian or even $\delta$-peak, always fourier decomposes into positive and negative frequencies and must therefore contain particle and antiparticle contributions: The energy disturbance through precise time measurement implies contributions of pair creation. Even if it is only measured whether the time of measurement is outside the lightcone or not, the above theorem in fourier theory tells, that all finite positive or negative energy intervals have nonvanishing amplitudes, thus one could not be certain whether positive energy, i.e. a particle, is found.

That there is no faster than light information transport in quantum field theory has been pointed out e.g. in [46]. As we saw, the spacelike propagation is merely a consequence of the uncertainty principle, and in general no quantum field theoretical propagators could be restricted to the inside of the lightcone. Independent of the actual dynamics and interactions, the propagators must have this feature. The deeper reason is, that due to the energy-time commutation relation the energy- and the time eigenbasis are related by fourier transformation which implies the well known uncertainty relation.

However, and this is now a new aspect, any generalisation of the Heisenberg algebra of 4-positions and 4-momenta will of course influence the propagator's behaviour through a modification of the uncertainty principle. Let us briefly discuss what can be expected from the introduction of nonzero minimal uncertainties in positions and momenta.

Nonzero minimal uncertainties in position and time measurements would provide an absolutely lower limit to the precision to which spacetime distances, and thus also light cones could be described. As we said, our uncertainty relation for a minimal position uncertainty has already appeared in the context of string theory, which is not unexpected since this is a theory of explicitly non-pointlike particles. A mini-

\footnotetext{
${ }^{9}$ More precisely, restricted to energies larger or equal to the restmass of the particle
} 
mal uncertainty in positions can also be motivated with considerations of gravity in the ultraviolet. The framework then expresses the old idea of a minimal observable length. For the propagators this should effectively imply an ultraviolet cutoff. We will here focus on the regularisation aspect.

On the other hand, our motivation is also to understand ordinary i.e. 'infrared' gravity, i.e. curvature as a source of nonzero minimal uncertainties in momentum and energy. It is easy to see, what it would mean if e.g. time dependent curvature implies nonzero minimal uncertainties in energy: The sharp distinction between positive frequencies (i.e. positive energies or particles travelling forward in time) and negative frequencies (i.e. negative energies or antiparticles travelling backwards in time) would no longer be possible. The propagators could reflect spontaneous creation of particles and energy nonconservation. On a general curved spacetime energy is of course not conserved and also spontaneous particle creation from gravity has been discussed in the literature, see e.g. [35, 36]. The concept of a particle as a irreducible representation of the Poincaré algebra can then no longer be applied straightforwardly. We will return to this question of curvature and the external symmetry group in Section 4.

Here we are not yet trying to make this contact to gravity explicit. Instead we will study how nonzero minimal uncertainties can be introduced into quantum field theory and whether this has a regularising effect. The basic idea of our approach is, to generalise the Heisenberg algebra of 4-positions and 4-momenta which also in quantum field theory 'sets the stage' of position and momentum space. Technically our proceeding is as follows:

While usually the path integral is performed over the space of fields where the fields are given in either the position or the momentum eigenbasis, we now rewrite the same path integral with the fields given in the Bargmann Fock basisto. Once the formalism is rewritten in the alternative basis we are independent of the classical position or momentum space. We do then no longer need or assume the existence of 4-position or 4-momentum eigenvectors. This allows us to generalise the Heisenberg algebra of the $\mathbf{x}_{\mu}$ and $\mathbf{p}_{\nu}$. Analogously to section 2, we can then abandon the notion of points in position or momentum space. Still all information on positions and momenta can be obtained from the matrix elements of the symmetric position and momentum operators.

We will have to address the important problem of defining the boundary conditions of the equations of motions in the generalised framework. The translation into Bargmann Fock space of the split-up of fields in their positive and negative frequency components, would not be difficult. However we would also have to find the correct generalisation of it for the noncommutative case. As we said, we expect this to be related e.g. to the Unruh effect. We postpone this discussion which would involve gravity. Let us in the beginning work with the euclidean signature instead.

\footnotetext{
${ }^{10}$ Recall that for the more general Heisenberg commutation relations the representations alternative to the $x$ or $p$ - representations are of course not necessarily Bargmann Fock representations.
} 
The generalisation of the commutation relations and of the formalism itself is then straightforward, also in four dimensions. On the other hand, when generalising Lagrangians, we will encounter the well known phenomenon that 'quantisation removes degeneracy' (Dirac): Once former commutative variables become noncommutative, there are then many different expressions which would all reduce to the same expression in the commutative case. One needs to develop a guiding principle to determine the 'right' generalised Lagrangians. We will only propose a working hypothesis. With the example of a quadratically divergent loop integration we then demonstrate that the introduction of the nonzero minimal uncertainties really has the power to regularise.

\subsection{Quantum field theory on Bargmann Fock space}

Using the path integral method we will now study the properties of the generalised field theoretical framework in the example of a charged scalar field with $\phi^{4}$ self interaction, in four euclidean dimensions. For the beginning we postpone the discussion of the noncommutative generalisation of the Feynman boundary conditions and work instead with the 4-dimensional euclidean signature where the boundary conditions are trivial $[$.

This standard theory we reformulate, so that the fields then live on Bargmann Fock space. Generalising Bargmann Fock space, we introduce nonzero minimal uncertainties in positions and momenta. We study whether this yields regularised behaviour of loop integrals. The simple example given is the divergent first order correction to the propagator.

\subsection{1 $\phi^{4}$-theory}

The generating functional for euclidean charged $\phi^{4}$ theory is

$$
Z\left[J, J^{*}\right]:=N \int D \phi D \phi^{*} e^{\int d^{4} x \phi^{*}\left(\partial_{i} \partial_{i}-\mu^{2}\right) \phi-\frac{\lambda}{4 !}(\phi \phi)^{*} \phi \phi+\phi^{*} J+J^{*} \phi}
$$

where velocities and actions are measured as multiples of $c$ and $\hbar$.

We reintroduce the fundamental constants:

$$
Z\left[J, J^{*}\right]=N \int D \phi D \phi^{*} e^{1 / \hbar \int d^{4} x \phi^{*}\left(\partial_{i} \partial_{i}-\frac{M^{2} c^{2}}{\hbar^{2}}\right) \phi-\frac{\lambda}{4 ! \hbar}(\phi \phi)^{*} \phi \phi+\phi^{*} J+J^{*} \phi}
$$

The fields and the coupling constant carry the units (e.g. in $\mathrm{kg}, \mathrm{m}, \mathrm{s}$ ):

$$
[\phi]=\sqrt{\frac{k g}{s}}, \quad[J]=\frac{1}{m^{2}} \sqrt{\frac{k g}{s}}, \quad[\lambda]=1
$$

\footnotetext{
${ }^{11}$ The reason being of course that the Wick rotation changes the hyperbolic equation of motion into an elliptic one, which then no longer has nontrivial homogeneous solutions.
} 
The theory is unchanged if we multiply the field by a constant. Using the (arbitrary) positive length $L$ we redefine: $\phi \rightarrow L \sqrt{\hbar} \phi:$

$$
Z\left[J, J^{*}\right]=N \int D \phi D \phi^{*} e^{\int d^{4} x \phi^{*}\left(-\frac{L^{2}}{\hbar^{2}} \mathbf{p}_{i} \mathbf{p}_{i}-\frac{L^{2} M^{2} c^{2}}{\hbar^{2}}\right) \phi-\frac{\lambda L^{4}}{4 !}(\phi \phi)^{*} \phi \phi+\phi^{*} J+J^{*} \phi}
$$

where we used $\mathbf{p}_{j} \phi(x):=-i \hbar \partial_{j} \phi(x)$. The units are now:

$$
[\phi]=1 / m^{2}, \quad[J]=1 / m^{2}, \quad[\lambda]=1
$$

This formulation is convenient for the transformation into the Bargmann Fock space, because as we will see it will keep the Bargmann Fock polynomials unitless.

\subsubsection{Fields on Bargmann Fock space}

Let us, term by term, transform the action functional into Bargmann Fock space:

The source terms are simply the scalar product of two functions:

$$
\begin{aligned}
\int d^{4} x \phi^{*}(x) J(x) & =\left.\overline{\phi(\bar{\eta})} e^{\partial_{\eta_{i}} \partial_{\bar{\eta}_{i}}} J(\bar{\eta})\right|_{0} \\
\int d^{4} x J^{*}(x) \phi(x) & =\left.\overline{J(\bar{\eta})} e^{\partial_{\eta_{i}} \partial_{\bar{\eta}_{i}}} \phi(\bar{\eta})\right|_{0}
\end{aligned}
$$

In the term quadratic in $\phi$ we recognise the scalar product of the function $\phi$ with the function $K . \phi$ where the operator $K$ is:

$$
\left.K=\left(-L^{2} / \hbar^{2}\right)\left(\mathbf{p}_{i} \mathbf{p}_{i}+M^{2} c^{2}\right) \quad \text { (summed over } i\right)
$$

While $\mathbf{p}_{j}$ would in momentum space be a multiplication operator, we know that it reads on Bargmann Fock space:

$$
\mathbf{p}_{j} \cdot \phi(\bar{\eta})=\frac{i \hbar}{2 L}\left(\bar{\eta}_{j}-\partial_{\bar{\eta}_{j}}\right) \phi(\bar{\eta})
$$

so that $K$ acts on functions on Bargmann Fock space as:

$$
-\frac{L^{2}}{\hbar^{2}}\left(\mathbf{p}_{i} \mathbf{p}_{i}+M^{2} c^{2}\right) \cdot \phi(\bar{\eta})=\left(\frac{1}{4} \sum_{i=1}^{4}\left(\bar{\eta}_{i}-\partial_{\bar{\eta}_{i}}\right)^{2}-\frac{L^{2} M^{2} c^{2}}{\hbar^{2}}\right) \phi(\bar{\eta})
$$

Thus, the quadratic term of the action reads:

$$
\begin{aligned}
& \int d^{4} x \phi^{*}(x)\left(-\frac{L^{2}}{\hbar^{2}} \mathbf{p}_{i} \mathbf{p}_{i}-\frac{L^{2} M^{2} c^{2}}{\hbar^{2}}\right) \phi(x) \\
&=\left.\overline{\phi(\bar{\eta})} e^{\partial_{\eta_{i}} \partial_{\bar{\eta}}{ }^{i}}\left(\frac{1}{4} \sum_{i=1}^{4}\left(\bar{\eta}_{i}-\partial_{\bar{\eta}_{i}}\right)^{2}-\frac{L^{2} M^{2} c^{2}}{\hbar^{2}}\right) \phi(\bar{\eta})\right|_{0}
\end{aligned}
$$


The interaction term is the scalar product of the function $\phi(x) \phi(x)$ with itself, where in $\phi(x) \phi(x)$ we recognise the pointwise multiplication $*$.

Thus the interaction term reads in Bargmann Fock space:

$$
-\frac{\lambda L^{4}}{4 !} \int d^{4} x \overline{\phi(x) \phi(x)} \phi(x) \phi(x)=-\left.\frac{\lambda L^{4}}{4 !} \overline{(\phi * \phi)(\bar{\eta})} e^{\partial_{\eta_{i}} \partial_{\bar{\eta}^{i}}}(\phi * \phi)(\bar{\eta})\right|_{0}
$$

where the pointwise multiplication $*$ is explicitly (from Eq.24):

$$
(\phi * \phi)(\bar{\eta})=\left.\frac{2}{3 \pi L^{2}} e^{\sum_{i=1}^{4}\left(\frac{1}{3}\left(\bar{\eta}_{i}+\partial_{\bar{\eta}_{i}^{\prime}}+\partial_{\bar{\eta}_{i}^{\prime \prime}}\right)^{2}-\frac{1}{2}\left(\bar{\eta}_{i}^{2}+\partial_{\bar{\eta}_{i}^{\prime}}^{2}+\partial_{\bar{\eta}_{i}^{\prime \prime}}^{2}\right)\right)} \phi\left(\bar{\eta}^{\prime}\right) \phi\left(\bar{\eta}^{\prime \prime}\right)\right|_{0}
$$

Note that the scale $L$ drops out when Eq.95 is put into Eq.94.

The use of the weighted pointwise multiplication $*^{\prime}$ instead of $*$, would lead to a modified infrared behaviour. In Bargmann Fock space it takes a simple form:

$$
\begin{gathered}
-\frac{\lambda L^{4}}{4 !} \int d^{4} x \overline{\phi(x) \phi(x)} \phi(x) \phi(x) e^{\frac{1}{2}\left(\frac{x}{L}\right)^{2}}=-\left.\frac{\lambda L^{4}}{4 !} \overline{\left(\phi *^{\prime} \phi\right)(\bar{\eta})} e^{\partial_{\eta_{i}} \partial_{\bar{\eta}}{ }^{i}}\left(\phi *^{\prime} \phi\right)(\bar{\eta})\right|_{0} \\
=\left.\frac{-\lambda}{4 \pi^{2} 4 !} \overline{\phi\left(\bar{\eta}+\partial_{\bar{\eta}^{\prime}}\right) \phi\left(\bar{\eta}+\bar{\eta}^{\prime}\right)} e^{\partial_{\eta_{i}} \partial_{\bar{\eta}}{ }^{i}} \phi\left(\bar{\eta}+\partial_{\bar{\eta}^{\prime \prime}}\right) \phi\left(\bar{\eta}+\bar{\eta}^{\prime \prime}\right)\right|_{0}
\end{gathered}
$$

Since $L$ can be chosen arbitrarily large, we could, up to the far infrared behaviour, approximate $*$ by the much easier to handle $*^{\prime}$.

The transformation into Bargmann Fock space is, like the transformation into e.g. momentum space, just a linear change of basis in the vector space of functions. The determinant of its Jacobian is a constant and can be absorbed in the overall factor of the path integral. We do thus not introduce anomalies.

\subsubsection{Feynman rules}

When working in the position or the momentum eigenbasis of the function space, we are formally dealing with an uncountably infinite number of basis states, namely the set of $\delta$-functions or the set of plane waves. However, the function space is actually separable. This means that there are bases of only a countable infinite number of vectors, which do also span the function space. The Bargmann Fock polynomials $\bar{\eta}^{m}$, which in position space are the Hermite functions, form such a countable basis, which spans the Hilbert space of square integrable functions (and in which we can expand even the non-normalisable $\delta$ - functions and plane waves, see Chapter 2).

Thus, we will now expand our $\phi$ in the discrete basis of Bargmann Fock polynomials $\bar{\eta}^{m}$. We will eventually express the action functional in terms of the coefficients that the vectors (i.e. the functions) and the operators have in this orthonormal basis:

$$
\phi(\bar{\eta})=\sum_{r_{1}, r_{2}, r_{3}, r_{4}=0}^{\infty} \phi_{\vec{r}} \frac{\bar{\eta}_{1}^{r_{1}} \bar{\eta}_{2}^{r_{2}} \bar{\eta}_{3}^{r_{3}} \bar{\eta}_{4}^{r_{4}}}{\sqrt{r_{1} ! r_{2} ! r_{3} ! r_{4} !}}
$$




$$
J(\bar{\eta})=\sum_{r_{1}, r_{2}, r_{3}, r_{4}=0}^{\infty} J_{\vec{r}} \frac{\bar{\eta}_{1}^{r_{1}} \bar{\eta}_{2}^{r_{2}} \bar{\eta}_{3}^{r_{3}} \bar{\eta}_{4}^{r_{4}}}{\sqrt{r_{1} ! r_{2} ! r_{3} ! r_{4} !}}
$$

Thus a function $\phi$ is now represented by its coefficient vector $\phi_{\vec{r}}=\phi_{r_{1}, r_{2}, r_{3}, r_{4}}$ with the indices $r_{i}=0,1,2, \ldots \infty,(i=1, \ldots, 4)$. We ordered the polynomials because, due to commutativity, e.g.

$$
\frac{\bar{\eta}_{1}^{r_{1}} \bar{\eta}_{2}^{r_{2}} \bar{\eta}_{3}^{r_{3}} \bar{\eta}_{4}^{r_{4}}}{\sqrt{r_{1} ! r_{2} ! r_{3} ! r_{4} !}}=\frac{\bar{\eta}_{4}^{r_{4}} \bar{\eta}_{2}^{r_{2}} \bar{\eta}_{3}^{r_{3}} \bar{\eta}_{1}^{r_{1}}}{\sqrt{r_{1} ! r_{2} ! r_{3} ! r_{4} !}}
$$

so that the set of all (nonordered) polynomials would have been overcomplete. In our generalised formalism, when the polynomials in $\bar{\eta}$ 's become noncommutative, the ordered polynomials will still form a basis ${ }^{\text {2Z }}$.

The coefficient matrix of the quadratic operator is then calculated as

$$
M_{\vec{r} \vec{s}}=\left.\frac{\overline{\bar{\eta}_{1}^{r_{1}} \bar{\eta}_{2}^{r_{2}} \bar{\eta}_{3}^{r_{3}} \bar{\eta}_{4}^{r_{4}}}}{\sqrt{r_{1} ! r_{2} ! r_{3} ! r_{4} !}} e^{\partial_{\eta_{i}} \partial_{\bar{\eta}}{ }^{i}}\left(-\frac{1}{4} \sum_{i=1}^{4}\left(\bar{\eta}_{i}-\partial_{\bar{\eta}_{i}}\right)^{2}+\frac{L^{2} M^{2} c^{2}}{\hbar^{2}}\right) \frac{\bar{\eta}_{1}^{s_{1}} \bar{\eta}_{2}^{s_{2}} \bar{\eta}_{3}^{s_{3}} \bar{\eta}_{4}^{s_{4}}}{\sqrt{s_{1} ! s_{2} ! s_{3} ! s_{4} !}}\right|_{0}
$$

and the interaction term has the coefficients:

$$
V_{\vec{t} \vec{u} \vec{v} \vec{w}}=\left.\frac{\bar{\eta}_{1}^{t_{1}} \bar{\eta}_{2}^{t_{2}} \bar{\eta}_{3}^{t_{3}} \bar{\eta}_{4}^{t_{4}} * \bar{\eta}_{1}^{t_{1}} \bar{\eta}_{2}^{t_{2}} \bar{\eta}_{3}^{t_{3}} \bar{\eta}_{4}^{t_{4}}}{\sqrt{t_{1} ! t_{2} ! t_{3} ! t_{4} ! u_{1} ! u_{2} ! u_{3} ! u_{4} !}} e^{\partial_{\eta_{i}} \partial_{\bar{\eta}_{i}}} \frac{\bar{\eta}_{1}^{v_{1}} \bar{\eta}_{2}^{v_{2}} \bar{\eta}_{3}^{v_{3}} \bar{\eta}_{4}^{v_{4}} * \bar{\eta}_{1}^{w_{1}} \bar{\eta}_{2}^{w_{2}} \bar{\eta}_{3}^{w_{3}} \bar{\eta}_{4}^{w_{4}}}{\sqrt{v_{1} ! v_{2} ! v_{3} ! v_{4} ! w_{1} ! w_{2} ! w_{3} ! w_{4} !}}\right|_{0}
$$

Note that the path integration can be written as the product of a countably infinite number of integrations:

$$
\begin{aligned}
\int D \phi D \phi^{*} e^{-S\left[\phi(x), \phi^{*}(x)\right]} & =N \int D \phi D \bar{\phi} e^{-S[\phi(\bar{\eta}), \bar{\phi}(\bar{\eta})]} \\
& =N \int \prod_{r_{1}, r_{2}, r_{3}, r_{4}=0}^{\infty} d \phi_{r_{1}, r_{2}, r_{3}, r_{4}} d \phi_{r_{1}, r_{2}, r_{3}, r_{4}}^{*} e^{-S\left[\phi_{\vec{r}}, \phi_{\vec{r}}^{*}\right]}
\end{aligned}
$$

The Feynman rules are derived in the standard way. The generating functional now reads:

$$
Z\left[J, J^{*}\right]=N \int D \phi D \phi^{*} e^{-\phi_{\vec{r}}^{*} M_{\vec{r} \vec{s}} \phi_{\vec{s}}+\phi_{\vec{r}}^{*} J_{\vec{r}}+J_{\vec{r}}^{*} \phi_{\vec{r}}-\frac{\lambda L^{4}}{4 !} V_{\vec{t} \vec{u} \vec{v} \vec{w}} \phi_{\vec{t}}^{*} \phi_{\vec{u}}^{*} \phi_{\vec{v}} \phi_{\vec{w}}}
$$

Here, each index vector denotes four indices, corresponding to the four euclidean dimensions, e.g. $\vec{r}=r_{1}, r_{2}, r_{3}, r_{4}$ where each index is summed over, e.g. $r_{2}=0,1,2, \ldots \infty$. Pulling the interaction term in front of the integral yields:

$$
Z\left[J, J^{*}\right]=N e^{-\frac{\lambda L^{4}}{4 !} V_{\vec{r} \vec{s} \vec{t}} \frac{\partial}{\partial J_{\vec{r}}} \frac{\partial}{\partial J_{\vec{s}}} \frac{\partial}{\partial J_{\vec{t}}} \frac{\partial}{\partial J_{\vec{u}}}} \int D \phi D \phi^{*} e^{-\phi_{\vec{r}}^{*} M_{\vec{r} \vec{s}} \phi_{\vec{s}}+\phi_{\vec{r}}^{*} J_{\vec{r}}+J_{\vec{r}}^{*} \phi_{\vec{r}}}
$$

\footnotetext{
${ }^{12}$ This requirement, i.e. technically the invariance of the Poincaré series (i.e. of the dimensionalities of the subspaces of polynomials of equal grade), was one of the key conditions in the derivation of the generalised commutation relations, see [9].
} 
We rearrange the remaining integrand:

$$
Z\left[J, J^{*}\right]=N e^{-\frac{\lambda L^{4}}{4 !} V_{\vec{r} \vec{s} \vec{t} \vec{u}} \frac{\partial}{\partial J_{\vec{r}}} \frac{\partial}{\partial J_{\vec{s}}} \frac{\partial}{\partial J_{\vec{t}}} \frac{\partial}{\partial J_{\vec{u}}}} \int D \phi D \phi^{*} e^{-\left(\phi_{\vec{r}}^{*}-J_{\vec{s}}^{*} M_{\vec{s} \vec{r}}^{-1}\right) M_{\vec{r}, \vec{t}}\left(\phi_{\vec{t}}-M_{\vec{t} \vec{u}}^{-1} J_{\vec{u}}\right)+J_{\vec{r}}^{*} M_{\vec{r} \vec{s}} J_{\vec{s}}}
$$

The path integral can now be absorbed in the overall constant:

$$
Z\left[J, J^{*}\right]=N^{\prime} e^{-\frac{\lambda L^{4}}{4 !} V_{\vec{r} \vec{s} \vec{t} \vec{u}} \frac{\partial}{\partial J_{\vec{r}}} \frac{\partial}{\partial J_{\vec{s}}} \frac{\partial}{\partial J_{\vec{t}}} \frac{\partial}{\partial J_{\vec{u}}}} e^{J_{\vec{r}}^{*} M_{\vec{r} \vec{s}} J_{\vec{s}}}
$$

For the Feynman rules we read off:

$$
\begin{aligned}
& \text { free propagator: } \Delta_{o}(\vec{a}, \vec{b})=M_{\vec{a} \vec{b}}^{-1} \\
& \text { vertex: } \quad \Gamma_{0}(\vec{a}, \vec{b}, \vec{c}, \vec{d})=-\frac{\lambda L^{4}}{4 !} V_{\vec{a} \vec{b} \vec{c} \vec{d}}
\end{aligned}
$$

\subsubsection{Loop integration}

The usual four dimensional loop integrations, e.g. over momentum space, now become four discrete summations.

For example, differentiating $Z\left[J, J^{*}\right]$ with respect to $J_{\vec{a}}^{*}$ and $J_{\vec{b}}$ at vanishing sources, and neglecting the disconnected terms, yields for the first propagator:

$$
\Delta(\vec{a}, \vec{b})=M_{\vec{a} \vec{b}}^{-1} \quad-4 \frac{\lambda L^{4}}{4 !} \cdot \sum_{r_{1}, r_{2}, r_{3}, r_{4}=0}^{\infty} \sum_{s_{1}, s_{2}, s_{3}, s_{4}=0}^{\infty} V_{\vec{a} \vec{r} \vec{r} \vec{b}} M_{\vec{a} \vec{r}}^{-1} M_{\vec{r} \vec{s}}^{-1} M_{\vec{s} \vec{b}}^{-1}+\ldots
$$

The correction is the tadpole term which in momentum space reads, up to the external legs and a constant,

$$
-\frac{\lambda}{6} \int d^{4} p \frac{1}{p_{i} p^{i}+M^{2} c^{2}}=\text { divergent }
$$

and which is a quadratic divergency. If we now calculated $M^{-1}$ and $V$ explicitly and applied them in Eq.109 we could only recover that the summations are divergent. Let us therefore turn to the generalised framework where we will work with the explicit matrices $M^{-1}$ and $V$ and find that this graph becomes regularised.

\subsection{Generalised quantum field theory on Bargmann Fock space}

Our strategy in generalising the quantum field theoretical framework was to first reformulate the path integral so that the fields live on Bargmann Fock, rather than

\footnotetext{
${ }^{13}$ Recall that one could formally avoid this tadpole diagram by, in the operator formalism, normal ordering the interaction lagrangian. This would amount to an (infinite) mass renormalisation.
} 
on position or momentum space. We now generalise the commutation relations of the position and momentum operators, completely like in section 2, only in four dimensions now. In this way we introduce nonzero minimal uncertainties in positions and momenta, and abandon position or momentum eigenfunctions. We can use the techniques developed in section 2 to transform the fields, operators and the scalar product into noncommutative Bargmann Fock space.

Thus the kinematics, i.e. the framework itself, can be generalised straightforwardly. However, the generalisation of the dynamics i.e. the generalisation of Lagrangians is not straightforward.

There are at least three different sources of ambiguity in generalising Lagrangians:

1. A generalised Lagrangian need not necessarily become the old Lagrangian as we remove the generalisation parameter (i.e. here as $q \rightarrow 1$ ). In this limit we need to recover the old theory only where the old theory is actually valid. This means, that the generalised Lagrangian may deviate in the far infrared or in the far ultraviolet, even as $q \rightarrow 1$. Ultimately we would hope that the correctly generalised Lagrangians allow for the description of the gravity effects that appear at these scales.

2. We need a generalised notion of pointwise multiplication. In order to describe local interactions, usual quantum field theoretical Lagrangians rely on the notion of pointwise multiplication $*$ of fields. Usually we can transform fields on Bargmann Fock space into position space, multiply them pointwise and transform the result back into Bargmann Fock space. In this way one obtains the pointwise multiplication of Bargmann Fock functions. However, in the generalised case there is no transformation into a position space any more because we abandon position eigenstates i.e. we abandon the very notion of a point in position space. Thus, in the generalised case the 'pointwise' multiplication of functions on Bargmann Fock space, can be defined quite arbitrarily. As one removes the generalisation parameter it has to reduce to ordinary pointwise multiplication but, as we said in 1, possibly only up to the far UV or IR.

3. 'Quantisation removes degeneracy' (Dirac) originally meant effects that appear as the phase space becomes noncommutative i.e. that appeared by quantisation. How would e.g. a function $f(x, p)$ (with $[x, p]=0$ ) read when the variables $x, p$ become noncommutative: $[x, p]=i \hbar$. There is then obviously no unique $f(x, p)$ due to ordering ambiguities. An analogous ambiguity appears here, as we generalise the commutation relations even further, so that also the position operators among themselves, as well as the momentum operators among themselves become noncommutative.

A guiding principle is needed that determines the 'correct' generalisation of Lagrangians. This guiding principle must of course be found on a physical basis. Ultimately, a 'correctly' generalised Lagrangian should allow to understand the generalisation parameters, more or less directly, as gravitational degrees of freedom. Large scale gravity i.e. general relativity is well confirmed. It must of course be studied whether 
in our approach one can at least reproduce this large scale gravity as an effective theory in the medium and large distances. Work in this direction is in progress. On the other hand, only little is known about gravity in the ultraviolet, i.e. at small distances like the Planck length. At this stage we can only speculate whether e.g. positions and momenta then play with reversed rôles, i.e. that then noncommutativity of the position operators becomes crucial (and may even reflect a 'curvature-like' feature of momentum space). The beautyful duality of the positions and momenta is usually thought to be restricted to the kinematics. The main reason is of course that interactions appear to be local only in position space, a fact which singles out position space. However, as we said, the very concept of locality needs to be reconsidered for a quantum theory that includes gravity. Our formalism offers a framework in which such new ideas can be formulated and studied.

We have not yet developed representations of our fully generalised commutation relations 72-74. We can therefore not yet work with the correspondingly generalised quantum field theoretical framework. However, we do have representations of two sub classes of our generalised commutation relations. We will study the generalisation of the quantum field theoretical framework for these examples. Once representations for the fully general commutation relations are found, it should be straightforward to also work out the correspondingly generalised quantum field theoretical framework.

As we said, a guiding principle for the generalisation of arbitrary Lagrangians is needed. We do yet attempt to propose such a guiding principle. Instead we will deal only with a working hypothesis for the generalisation of the Lagrangian of the bosonic scalar charged particle with self interaction. We focus on the technical study whether ultraviolet divergencies can become regularised through nonzero minimal uncertainties.

\subsubsection{Generalised $\phi^{4}$ theory}

We study features of the generalised quantum field theoretical framework in the simple example of the $\phi^{4}$ theory. The formulas for our first sub class of generalised commutation relations will be given explicitly. From these, the corresponding expressions for the case of our second sub class of commutation relations can easily be derived. In particular, our main result, namely the convergence of our example graph, will be derived for both cases.

The original generating functional, with the fields defined on position space, reads:

$$
Z\left[J, J^{*}\right]=N \int D \phi D \phi^{*} e^{\int d^{4} x \phi^{*}\left(-\frac{L^{2}}{\hbar^{2}} p_{i} p_{i}-\frac{L^{2} M^{2} c^{2}}{\hbar^{2}}\right) \phi-\frac{\lambda L^{4}}{4 !}(\phi \phi)^{*} \phi \phi+\phi^{*} J+J^{*} \phi}
$$

The generalised Lagrangian need not to reduce exactly to the above Lagrangian in the limit $q \rightarrow 1$. As we said, we would allow the generalised Lagrangian to deviate as $q \rightarrow 1$ from Eq.111 in the IR and UV, since the ordinary theory does not describe the gravity effects in the large and in the very small distances. 
Let us propose the following working hypothesis:

We will use the freedom in the generalisation to work with a generalised Lagrangian that, as $q \rightarrow 1$, reduces to the ordinary $\phi^{4}$ Lagrangian, but deviates in the infrared behaviour. We introduce a modified long distance behaviour of the quadratic term, which is mathematically quite natural and one could even speculate about a possible physical justification. Our modification of the infrared behaviour of also the interaction term has its reason only in that this modified interaction term is more straightforward to generalise and easier to handle than the original interaction term.

We do not claim that this is physically sensible. Our main objective here, is to study our generalised quantum field theoretical framework with the example of a toy Lagrangian that is mathematically easy to deal with.

Explicitly our working hypothesis for the generalised $\phi^{4}$ theory is:

$$
\begin{aligned}
Z\left[J, J^{*}\right]=N \int D \phi D \phi^{*} & e^{\left.\overline{\phi(\bar{\eta})} e_{1 / q}^{\partial \eta_{i} \partial \bar{\eta}_{i}}\left(-\bar{\eta}_{i} \partial_{\bar{\eta}_{i}}-2-\frac{L^{2} M^{2} c^{2}}{\hbar^{2}}\right) \phi(\bar{\eta})\right|_{0}} \\
\cdot & e^{-\left.\frac{\lambda}{4 \pi^{2} 4 !} \overline{\phi\left(\bar{\eta}+\partial_{\bar{\eta}^{\prime}}\right) \phi\left(\bar{\eta}+\bar{\eta}^{\prime}\right)} e_{1 / q}^{\partial \eta_{i} \partial_{\bar{\eta}_{i}}} \phi\left(\bar{\eta}+\partial_{\bar{\eta}^{\prime \prime}}\right) \phi\left(\bar{\eta}+\bar{\eta}^{\prime \prime}\right)\right|_{0}} \\
\cdot & e^{\left.\overline{\phi(\bar{\eta})} e_{1 / q}^{\partial \eta_{i} \partial \bar{\eta}_{i}} J(\bar{\eta})\right|_{0}+\left.\overline{J(\bar{\eta})} e_{1 / q}^{\partial \eta_{i} \partial_{\bar{\eta}_{i}}} \phi(\bar{\eta})\right|_{0}}
\end{aligned}
$$

It is clear that our Lagrangian reduces, for $q \rightarrow 1$, and then transformed into position space, to the Lagrangian:

$$
\mathcal{L}^{\prime}=\phi^{*}\left(-\frac{L^{2}}{\hbar^{2}} \mathbf{p}_{i} \mathbf{p}_{i}-\frac{\mathbf{x}_{i} \mathbf{x}_{i}}{4 L^{2}}-\frac{L^{2} M^{2} c^{2}}{\hbar^{2}}\right) \phi-\frac{\lambda L^{4}}{4 !}(\phi \phi)^{*} \phi \phi e^{\frac{x_{i} x_{i}}{2 L^{2}}}+\phi^{*} J+J^{*} \phi
$$

Our change in the infrared behaviour of the interaction term is, to work with the pointwise multiplication $*^{\prime}$ instead of $*$. The change of the long distance behaviour in the kinetic term is the addition of the term $\mathbf{x}_{i} \mathbf{x}_{i} / 4 L^{2}$. We thus work with the operator $\bar{\eta}_{i} \partial_{\bar{\eta}_{i}}$ which is diagonal in the Bargmann Fock basis, so that also the free propagator will be diagonal. Physically, we are treating the free particle as a particle in a harmonic oscillator potential with the backdriving force negligibly small up to large distances (we assume $L$ large). This of course means that we are breaking the 10- dimensional Poincaré symmetry down to the 6- dimensional Lorentz symmetry. However, the Lorentz symmetry $S O(3,1)$ is actually enlarged to the unitary group $S U(3,1)$ which has 15 , i.e. even more dimensions than the Poincaré group. On the other hand, the appearance of a harmonic oscillator potential from gravity seems not very likely. Note however that e.g. a test particle inside a homogeneous, spherical symmetric gravitating energy distribution experiences gravity as a harmonic oscillator potential. (This is e.g. how a particle inside the earth experiences the earth's gravity.)

Let us however stress that these arguments only apply to the toy model that we are considering here. The correct generalisation of the dynamics and of the Poincaré algebra will go beyond the quantum group symmetric case and can of course only be

\footnotetext{
${ }^{14}$ The $S U(3,1)$ has a quantum group generalisation $S U_{q}(3,1)$, see $[5]$.
} 
obtained from a detailed analysis of the physical origin of the modifications to the commutation relations. We will begin this discussion in Section 4.

Our working hypothesis for the generalised Lagrangian reduces, as $q \rightarrow 1$ to the ordinary $\phi^{4}$ Lagrangian for short distances, i.e. in the ultraviolet. We will see in an example that our generalisation of the framework itself, i.e. of the commutation relations and thus the introduction of nonzero minimal uncertainties in positions and momenta, does affect e.g. the ultraviolet in a regularising way.

\subsubsection{Fields on Bargmann Fock space}

The path integral in Eq.112 is straightforward to evaluate, whether $q=1$ or $q>1$ : Summing over all fields means to sum over all Bargmann Fock functions, i.e. to sum over all distinct polynomials (or power series) in the $\bar{\eta}$ 's. Each such Bargmann Fock function is to be put into the exponentiated action functional in order to obtain its amplitude. For clearity let us consider simple examples:

The simplest Bargmann Fock function is the constant $\phi(\bar{\eta}):=1$. We calculate the amplitude i.e. the contribution to the path integral Eq.112 of $\phi(\bar{\eta})=1=\overline{\phi(\bar{\eta})}$. From the source terms we get:

$$
\begin{aligned}
& e^{\left.1 e_{1 / q}^{\partial \eta_{i} \partial \bar{\eta}_{i}} J(\bar{\eta})\right|_{0}+\left.\overline{J(\bar{\eta})} e_{1 / q}^{\partial \eta_{i} \partial_{\bar{\eta}_{i}}} 1\right|_{0}} \\
= & e^{\left.1 \sum_{r=0}^{\infty} \frac{\partial \eta_{i} \partial \bar{\eta}_{i}}{[r] !} J(\bar{\eta})\right|_{\eta=0, \bar{\eta}=0}+\left.\overline{J(\bar{\eta})} \sum_{r=0}^{\infty} \frac{\partial_{\eta_{i}} \partial_{\bar{\eta}_{i}}}{[r] !} 1\right|_{\eta=0, \bar{\eta}=0}} \\
= & e^{J_{0,0,0,0}+J_{0,0,0,0}^{*}}
\end{aligned}
$$

where we expanded the source in the basis of the ordered orthonormal polynomials:

$$
J(\bar{\eta})=\sum_{s_{1}, s_{2}, s_{3}, s_{4}=0}^{\infty} J_{s_{1} s_{2} s_{3} s_{4}} \frac{\bar{\eta}_{1}^{s_{1}} \bar{\eta}_{2}^{s_{2}} \bar{\eta}_{3}^{s 3} \bar{\eta}_{4}^{s_{4}}}{\sqrt{\left[s_{1}\right] !\left[s_{2}\right] !\left[s_{3}\right] !\left[s_{4}\right] !}}
$$

This expansion of an arbitrary $J$ is possible because even now that the $\bar{\eta}$ 's are noncommutative, we can still rewrite each polynomial in the ordered form, see [9]. Recall that in general the evaluation procedure is to first commute all $\partial_{\eta}$ 's to the left and all $\partial_{\bar{\eta}}$ 's to the right, using the (generalised) Leibniz rules and commutation relations among the $\bar{\eta}$ 's, among the $\partial_{\bar{\eta}}$ 's and their complex conjugates. The evaluation is to be carried out at $\eta=0$ and $\bar{\eta}=0$ i.e. we keep only the constant terms and set all other terms equal zero.

In the term quadratic in $\phi$ and in the interaction term the derivatives of the $q$ exponential do now not find $\bar{\eta}$ 's to act on, so that we can immediately evaluate. Thus the amplitude for $\phi(\bar{\eta})=1, \overline{\phi(\bar{\eta})}=1$ is:

$$
\operatorname{ampl}\left[\phi(\bar{\eta})=1, \overline{\phi(\bar{\eta})}=1, J, J^{*}\right]=e^{-2-\frac{L^{2} M^{2} c^{2}}{\hbar^{2}}-\frac{\lambda}{4 \pi^{2} 4 !}+J_{0,0,0,0}+J_{0,0,0,0}^{*}}
$$


In the calculation of the amplitude for e.g. $\phi(\bar{\eta}):=\bar{\eta}_{3}, \overline{\phi(\bar{\eta})}=\eta_{3}$ there are now some differentiations to be evaluated. E.g. the contribution of the interaction term is:

$$
\begin{aligned}
& -\left.\frac{\lambda}{4 \pi^{2} 4 !} \overline{\left(\bar{\eta}_{3}+\partial_{\bar{\eta}_{3}^{\prime}}\right)\left(\bar{\eta}_{3}+\bar{\eta}_{3}^{\prime}\right)} e_{1 / q}^{\partial_{\eta_{i}} \partial_{\bar{\eta}_{i}}}\left(\bar{\eta}_{3}+\partial_{\bar{\eta}_{3}^{\prime}}\right)\left(\bar{\eta}_{3}+\bar{\eta}_{3}^{\prime}\right)\right|_{0} \\
= & -\left.\frac{\lambda}{4 \pi^{2} 4 !}\left(\eta_{3}^{2}+1\right) e_{1 / q}^{\partial_{\eta_{i}} \partial_{\bar{\eta}_{i}}}\left(\bar{\eta}_{3}^{2}+1\right)\right|_{0} \\
= & -\frac{\lambda}{4 \pi^{2} 4 !}([2]+1)=-\frac{\lambda}{4 \pi^{2} 4 !}\left(q^{2}+2\right)
\end{aligned}
$$

The amplitude is then easily found to be:

$$
\operatorname{ampl}\left[\phi(\bar{\eta})=\bar{\eta}_{3}, \overline{\phi(\bar{\eta})}=\eta_{3}, J, J^{*}\right]=e^{-1-2-\frac{L^{2} M^{2} c^{2}}{\hbar^{2}}-\frac{\lambda}{4 \pi^{2} 4 !}\left(q^{2}+2\right)+J_{0,0,1,0}+J_{0,0,1,0}^{*}}
$$

In this way the amplitudes for arbitrary $\phi, \bar{\phi}$ can be calculated: In the action functional, using the commutation relations, the $\partial_{\eta}$ 's and $\partial_{\bar{\eta}}$ 's are commuted to the left and right. We evaluate at 0 i.e. we keep only the constants. This yields a number, the exponential of which is the amplitude that corresponds to the field considered.

The integrations over position or momentum space have become integrations over Bargmann Fock space, which can be written in terms of algebraic differentiations and summations. The $n$ - point functions $\Gamma$ now have their arguments in Bargmann Fock space rather than in position or momentum space. Nevertheless all information on positions and momenta can still be obtained.

In the ordinary framework the unitary transformations given in section 2 could be used to transform the propagators, vertices, $n$-point functions or $S$-matrix elements into momentum or position space. Let us consider e.g. a field given as a Bargmann Fock function $\phi(\bar{\eta})$. In order to obtain its component $\phi\left(p^{\prime}\right)$ of a specific 4-momentum $p^{\prime}$ one simply calculates (in Bargmann Fock space) the scalar product of the function $\phi(\bar{\eta})$ with the momentum eigenvector $\phi_{p^{\prime}}(\bar{\eta})$. The form of the momentum eigenvectors in Bargmann Fock space can either be calculated directly in Bargmann Fock space or can also easily be obtained from its form in the position or in the momentum eigenbasis, using e.g. Eq.13. In the generalised framework there are no more momentum (or position) eigenvectors in the representation of the Heisenberg algebras. We can however still calculate the scalar product with e.g. those Bargmann Fock polynomials which describe particles that are relatively localised in momentum (or position) space, but of course not more precisely than $\Delta p_{0}$ or $\Delta x_{0}$. The calculation of these approximating polynomials was discussed in section 2.2 .3 .

\subsubsection{Feynman rules}

Like in Sec. 3.1.3 it is more convenient for the derivation of the Feynman rules to work simply with the coefficients of the fields in the orthonormal basis of ordered 
polynomials:

$$
\phi(\bar{\eta})=\sum_{s_{1}, s_{2}, s_{3}, s_{4}=0}^{\infty} \phi_{s_{1} s_{2} s_{3} s_{4}} \frac{\bar{\eta}_{1}^{s_{1}} \bar{\eta}_{2}^{s_{2}} \bar{\eta}_{3}^{s_{3}} \bar{\eta}_{4}^{s_{4}}}{\sqrt{\left[s_{1}\right] !\left[s_{2}\right] !\left[s_{3}\right] !\left[s_{4}\right] !}}
$$

In this basis the generating functional has the form:

$$
Z\left[J, J^{*}\right]=N \int D \phi D \phi^{*} e^{-\phi_{\vec{r}}^{*} M_{\vec{r} \vec{s}} \phi_{\vec{s}}+\phi_{\vec{r}}^{*} J_{\vec{r}}+J_{\vec{r}}^{*} \phi_{\vec{r}}-\frac{\lambda}{4 \pi^{2} 4 !} V_{\vec{t} \vec{u} \vec{w}} \phi_{\vec{t}}^{*} \phi_{\vec{u}}^{*} \phi_{\vec{v}} \phi_{\vec{w}}}
$$

The term quadratic in $\phi$, i.e. the matrix $M$ is actually diagonal:

$$
M_{\vec{r} \vec{s}}=\left(\left[r_{1}+r_{2}+r_{3}+r_{4}\right]+2+\frac{L^{2} M^{2} c^{2}}{\hbar^{2}}\right) \delta_{\vec{r}, \vec{s}}
$$

The usual derivation of the Feynman rules goes through and we obtain the free propagator:

$$
\Delta_{0}(\vec{r}, \vec{s})=\frac{1}{\left[r_{1}+r_{2}+r_{3}+r_{4}\right]+2+\frac{L^{2} M^{2} c^{2}}{\hbar^{2}}} \delta_{\vec{r}, \vec{s}}
$$

We calculate the simplest matrix elements of the vertex:

$$
\begin{aligned}
\Gamma_{0}(\overrightarrow{0}, \vec{r}, \overrightarrow{0}, \vec{s}) & =\Gamma_{0}(\vec{r}, \overrightarrow{0}, \overrightarrow{0}, \vec{s})=\Gamma_{0}(\overrightarrow{0}, \vec{r}, \vec{s}, \overrightarrow{0})=\Gamma_{0}(\vec{r}, \overrightarrow{0}, \vec{s}, \overrightarrow{0})= \\
& =-\frac{\lambda}{4 \pi^{2} 4 !} V_{\overrightarrow{0} \vec{r} \overrightarrow{0} \vec{s}}=\ldots=-\frac{\lambda}{4 \pi^{2} 4 !} \delta_{\vec{r}, \vec{s}}
\end{aligned}
$$

Clearly all other matrix elements of the vertex are also finite.

\subsubsection{Loop integration}

Let us now study the example of the first order correction to the propagator, namely the truncated tadpole graph. In the usual, unregularised theory it is quadratically divergent, see Eq.110. We calculate the off shell amplitude $\Sigma(\overrightarrow{0}, \overrightarrow{0})$ for an in- and an out-going Gaußian wavepacket $\phi(\bar{\eta})=1$. From the usual perturbative expansion

$$
\Delta(\vec{a}, \vec{b})=M_{\vec{a} \vec{b}}^{-1} \quad-\frac{\lambda}{\pi^{2} 4 !} \cdot \sum_{r_{1}, r_{2}, r_{3}, r_{4}=0}^{\infty} \sum_{s_{1}, s_{2}, s_{3}, s_{4}=0}^{\infty} V_{\vec{a} \vec{r} \vec{b} \vec{b}} M_{\vec{a} \vec{r}}^{-1} M_{\vec{r} \vec{s}}^{-1} M_{\vec{s} \vec{b}}^{-1}+\ldots
$$

this amplitude is:

$$
\begin{aligned}
\Sigma(\overrightarrow{0}, \overrightarrow{0}) & =-\frac{\lambda}{4 \pi^{2} 4 !} \cdot \sum_{r_{1}, r_{2}, r_{3}, r_{4}=0}^{\infty} \sum_{s_{1}, s_{2}, s_{3}, s_{4}=0}^{\infty} V_{\overrightarrow{0} \vec{r} \overrightarrow{0} 0} M_{\vec{r} \vec{s}}^{-1} \\
& =-\frac{\lambda}{\pi^{2} 4 !} \sum_{r_{1}, r_{2}, r_{3}, r_{4}=0}^{\infty} \frac{1}{\left[r_{1}+r_{2}+r_{3}+r_{4}\right]+2+\frac{L^{2} M^{2} c^{2}}{\hbar^{2}}} \\
& =-\frac{\lambda}{\pi^{2} 4 !} \sum_{r_{1}, r_{2}, r_{3}, r_{4}=0}^{\infty} \frac{1}{\frac{q^{2\left(r_{1}+r_{2}+r_{3}+r_{4}\right)-1}}{q^{2}-1}+2+\frac{L^{2} M^{2} c^{2}}{\hbar^{2}}}
\end{aligned}
$$


This is actually finite, i.e. $|\Sigma(\overrightarrow{0}, \overrightarrow{0})|<\infty$, because for large $r_{i}$ the sum goes like

$$
\left(q^{2}-1\right) \sum_{r_{1}, r_{2}, r_{3}, r_{4}=0}^{\infty} q^{-2\left(r_{1}+r_{2}+r_{3}+r_{4}\right)}
$$

which is the product of four geometrical series:

$$
=\left(q^{2}-1\right)\left(\frac{1}{1-q^{-2}}\right)^{4}
$$

Thus, the quadratic ultraviolet divergency has become a regularised function in the generalisation parameter $q$. It has, of course, a pole at $q=1$.

Since we had not explicitly modified the ultraviolet behaviour of the Lagrangian, we interpret this result such that this ultraviolet regularisation is built into the generalised framework itself.

Let us check whether our second sub class of generalised Heisenberg commutation relations also leads to the regularisation of this graph. It is not difficult to see that the construction of the generalised $\phi^{4}$ theory goes through straightforwardly. We now have to check the convergence of the loop summation:

$$
\sum_{r_{1}, r_{2}, r_{3}, r_{4}=0}^{\infty} \frac{1}{\left[r_{1}\right]_{q_{1}}+\left[r_{2}\right]_{q_{2}}+\left[r_{3}\right]_{q_{3}}+\left[r_{4}\right]_{q_{4}}+2+\frac{L^{2} M^{2} c^{2}}{\hbar^{2}}}
$$

To see that this is convergent, note first that it can be majorised by

$$
\sum_{r_{1}, r_{2}, r_{3}, r_{4}=0}^{\infty} \frac{1}{\left[r_{1}\right]_{q^{\prime}}+\left[r_{2}\right]_{q^{\prime}}+\left[r_{3}\right]_{q^{\prime}}+\left[r_{4}\right]_{q^{\prime}}+2+\frac{L^{2} M^{2} c^{2}}{\hbar^{2}}}
$$

where $q^{\prime}$ is the smallest of the $q_{i}$ (recall that all $q_{i}>1$ ). We now use the 'rotational' symmetry in the discrete summation space $\mathbf{N}^{4}$ of the $r_{i}$ to majorise this multiple sum by a simpler sum of the form

$$
\sum_{r=0}^{\infty} V(r) \frac{1}{q^{r}+\text { const }}
$$

Here $V(r)$ is number of terms that are to be summed over in the 'layer' determined by $r^{2} \leq \sum_{i=1}^{4} r_{i}^{2} \leq(r+1)^{2}$, which is essentially proportional to $r^{3}$. Thus, since the denominator in the sum Eq.126 grows exponentially, a simpe ratio test proves the convergence of our sum.

We do not have a proof that all the generalised commutation relations that imply nonzero minimal uncertainties lead in general to the convergence of loop graphs. The above results however appear to support the intuitive expectation that the introduction of nonzero minimal uncertainties does lead to effective cutoffs. 


\section{On the Poincaré algebra and the origins of min- imal uncertainties}

The Poincaré group plays a fundamental rôle for the particle concept in quantum field theory. A detailed study of the external 'symmetry' will therefor be needed for our generalised framework in order to properly define spin and statistics in the generalised setting, and also in order to check fundamental properties like microcausality and unitarity. We can not yet answer these important questions here, let us however indicate a possible start for these studies.

We note first that if a generalised Poincaré algebra, generated by rotations and translations can be defined in the generalised case, it should still be contained in the Heisenberg algebra. We will therefore first have to clarify if and how a physical origin of the generalisation Eqs.72-74 of the commutation relations of the Heisenberg algebra can be made precise.

\subsection{Ultraviolet generalisation of the uncertainty relations}

Let us mention that the way in which nonzero minimal uncertainties in positions enter our framework appears to be in agreement with results of quantum gravity and string theory, see [40, 41, 42, 43], where effective uncertainty relations have been derived, which are very similar to our uncertainty relations (Eq.66 for $\Delta p_{0}=0$ ), namely

$$
\Delta x \geq \frac{\hbar}{\Delta p}+\text { const } \cdot \Delta p
$$

On the other hand this is probably the simplest modification of the uncertainty relation that implies nonzero minimal uncertainties in positions. It is therefore quite likely to appear, at least as an approximation, in any theory of non-pointlike particles. Nevertheless, the coincidence of these results may also indicate that our framework could be used as an effective theory of an underlying theory of explicitly non-pointlike particles like string theory.

More general than the above one-dimensional uncertainty relation Eq.127 which is found in the literature, there appear in our $n$ - dimensional generalised commutation relations Eqs.7274 general matrices $B$ and $C$ which are related to nonzero minimal uncertainties in positions and further to a possible noncommutativity of the position operators among themselves. The introduction of such a matrices $B, C$ should be related to effects at extremely short distances like the Planck scale. We note that our general case is of the type that is given in the literature for the one-dimensional case, but we will here not speculate any further. In particular we can not yet discuss implications of such ultraviolet modifications on the external symmetry. 


\subsection{Infrared generalisation of the uncertainty relations}

In our generalised commutation relations Eqs.72-74 the matrices $A$ and $D$ have the potential to introduce nonzero minimal uncertainties in momenta and noncommutativity of the momentum operators among themselves. We expected such large scale or 'infrared' effects to occur from ordinary large scale gravity i.e. from curvature. We sketch an ansatz that motivates the occurrence of a matrix $A$ from large scale gravity:

Let us first explain why we intuitively expect curvature to produce nonzero minimal uncertainties in momenta or why it should at least modify the uncertainty relations: Quantum theoretically, as one tries to measure momentum more precisely, a particle's position gets more uncertain. Momentum is a nonlocal property of a particle. Measuring a momentum vector, in which vector space, i.e. in the tangent space to which point of the manifold should it live? Doing quantum mechanics on flat space, one can identify all tangent spaces, however this is no longer so in the presence of curvature due to the path-dependence of parallel transport. This should have an effect which becomes the more significant, the more the wave function is spread over spacetime. Intuitively we therefore expect this to yield a lower limit to the precision of momentum determination. In order to make this precise, we try an ansatz for how an observer $A$ at a point $Q$ on a curved space could define position and momentum operators, that would allow him to do quantum theory in the vicinity of $Q$, including e.g. first order curvature effects. Our strategy is, to relate position- or momentum measurements to vectors in the tangent- or cotangent space of $Q$.

The observer $A$ at a point $Q$ in spacetime may set up a geodesic coordinate system $G_{Q}$ with the event $Q$ at its origin. A can assign each event its 4-position coordinates with respect to this frame. But how should $A$ define 4 -momenta? Classically as well as quantum theoretically, momentum is the generator of translations, which means in practice that it can be determined by measuring infinitesimal distances (multiplied with the rest mass and divided by the infinitesimal eigentime).

In curved space too, let $A$ define momentum as the generators of translations. But how can $A$ measure (infinitesimal) distances? Just like in flat space, let $A$ define the distance of two events E1 and E2 to be that 4-vector $\alpha \in T_{Q}$, by which he has to (infinitesimally) move from $Q$ to $Q^{\prime}$, so that when setting up a parallel geodesic frame $G_{Q^{\prime}}$ there, the coordinates of $\mathrm{E} 1$ in $Q 1$ and $\mathrm{E} 2$ in $Q 2$ coincidet.

Let us calculate the transformation of position coordinates under the change to the geodesic coordinate frame $G_{Q}$ from another 'infinitesimally by $\alpha$ translated' geodesic frame $G_{Q^{\prime}}$, the axes of which start off parallel to the axes of $G_{Q}$. The origin $Q^{\prime}$ of the frame $G_{Q^{\prime}}$ has the coordinates $\alpha^{\mu}$ in the frame $G_{Q}$. Trivially still the coordinates

\footnotetext{
${ }^{15}$ Note that this yields the definition of momentum as generating passive translations. Active translations, 'moving all events by $\alpha$ within one coordinate frame' would not be well defined since the parallel transport of $\alpha$ to these events would depend on the path of the parallel transport, a fact which exactly expresses the presence of curvature
} 
$x^{\prime \mu}$ of events in the infinitesimal neighbourhood of $Q^{\prime}$ (i.e. all $x^{\prime \mu} x^{\prime \nu}=0$ ) transform into the frame $G_{Q}$ as $x^{\mu}=\alpha^{\mu}+x^{\prime \mu}$. For events further away from the origin the transformation is nontrivial since the (geodesic) axes of $G_{Q}$ and $G_{Q^{\prime}}$ are then no longer parallel. Nevertheless all event's (passive) coordinate transformations are well defined. We will here calculate this transformation to the second order $x^{\prime \mu} x^{\prime \nu}$ in the distance from the origin, which is of course the first order of length that includes curvature.

Let us recall the construction of a geodesic frame or of a 'Riemann normal coordinate system' around the point $Q$. Using the exponential mapping each vector in the tangent space of $Q$ is mapped to a point of the manifold near $Q$. The coordinates that we give this point are just the coordinates of this tangent vector in an orthonormal basis. Practically this means the following: Let us denote the coordinates of a tangent vector by $x^{\mu}$. Which point in the manifold has thus the coordinates $x^{\mu}$ ? It is the endpoint of the geodesic which starts at $Q$ with the initial velocity given by the tangent vector $x^{\mu}$ and evolved until the geodesic's parameter reaches 1 .

Clearly in a geodesic frame the geodesics through the origin are straight lines. E.g. the geodesic which runs from the point $Q^{\prime}$ with an initial velocity $x^{\prime \mu}$ to the event $E$ with the coordinates $x^{\prime \mu}$ (where its geodesic parameter reaches 1 ) is a straight line in the frame $G_{Q^{\prime}}$. We want to calculate $E$ 's coordinates in the frame $G_{Q}$ from its coordinates $x^{\prime \mu}$ in the frame $G_{Q^{\prime}}$. To this end we now simply calculate in the frame $G_{Q}$ the endpoint of this same geodesic which starts at $Q^{\prime}$ with the initial velocity $x^{\prime \mu}$, evolved until the geodesic's parameter reaches 1 . This is now nontrivial since our geodesic is not running through the origin of $Q$ and is therefore not a straight line in the frame $G_{Q}$. Taking into account the first deviations from the straight line, which occur at second order distance from $Q^{\prime}$ we obtain the following result:

The coordinates $x^{\mu}$ in the frame $G_{Q}$ of an event which has the coordinates $x^{\prime \mu}$ in the frame $G_{Q^{\prime}}$ read to second order in $x^{\prime}$ :

$$
x^{\mu}=\alpha^{\mu}+x^{\prime \mu}-\frac{1}{2} \Gamma_{\alpha \beta, \nu}^{\mu}(Q) \alpha^{\nu} x^{\prime \alpha} x^{\prime \beta}
$$

To see this, recall that $\Gamma(Q)=0$ in the frame $G_{Q}$ so that in this frame the geodesics through the point $Q^{\prime}$ (which define the frame $G_{Q^{\prime}}$ ) obey, to the given order, the equation:

$$
\frac{d^{2} x^{\mu}}{d s^{2}}+\Gamma_{\alpha \beta, \nu}^{\mu}(Q) \alpha^{\nu} \frac{d x^{\alpha}}{d s} \frac{d x^{\beta}}{d s}=0
$$

So far our definition of momentum as generating infinitesimal (passive) translations was classical. The observer $A$ at $Q$ can now also define the corresponding quantum theoretical momentum generators $\mathbf{p}_{\mu}$ namely such, that they generate just the

\footnotetext{
${ }^{16}$ In general this mapping is unique as far as the geodesics that start at $Q$ do not intersect.
} 
translations given by Eq.128, i.e. we require (recall that $\alpha$ is infinitesimal i.e. $\alpha^{2}=0$ )

$$
\begin{aligned}
e^{-\frac{\alpha^{\nu} \mathbf{p}_{\nu}}{i \hbar}} \mathbf{x}^{\mu} e^{\frac{\alpha^{\nu} \mathbf{p}_{\nu}}{i \hbar}} & =\mathbf{x}^{\mu}+\frac{1}{i \hbar} \alpha^{\nu}\left[\mathbf{x}^{\mu}, \mathbf{p}_{\nu}\right] \\
& =\mathbf{x}^{\mu}+\alpha^{\mu}-\frac{1}{2} \Gamma_{\alpha \beta, \nu}^{\mu}(Q) \alpha^{\nu} \mathbf{x}^{\alpha} \mathbf{x}^{\beta}
\end{aligned}
$$

which means that the position and momentum operators of our observer $A$ at the point $Q$ obey, to the first gravity including order, the commutation relations:

$$
\left[\mathbf{x}^{\mu}, \mathbf{p}_{\nu}\right]=i \hbar \delta_{\nu}^{\mu}-\frac{i \hbar}{4} \Gamma_{\alpha \beta, \nu}^{\mu}(Q)\left\{\mathbf{x}^{\alpha}, \mathbf{x}^{\beta}\right\}
$$

Recall that the $\mathbf{x}^{\mu}$ are not the coordinate functions $x^{\mu}$, which are not contravariant vectors. The newly defined $\mathbf{x}^{\mu}$ and $\mathbf{p}_{\nu}$ are the components of covariant and contravariant vectors at $Q$, which generate an abstract Heisenberg algebra attached to $Q$ and which is to be represented on a Hilbert space. We have to check that our definition is covariant.

Actually, at the origin of geodesic coordinate systems, i.e. here at $Q$, there holds [49]:

$$
\Gamma_{\alpha \beta, \nu}^{\mu}(Q)=-\frac{1}{3}\left(R_{\alpha \beta \nu}^{\mu}+R_{\beta \alpha \nu}^{\mu}\right)
$$

Using the antisymmetry of the Riemann curvature tensor $R$ in its last two indices and its relation to the Jacobi curvature tensor $J$

$$
J_{\nu \alpha \beta}^{\mu}=\frac{1}{2}\left(R_{\alpha \nu \beta}^{\mu}+R_{\beta \nu \alpha}^{\mu}\right)
$$

we find that the set of commutation relations is covariant and has the simple form:

$$
\left[\mathbf{x}^{\mu}, \mathbf{p}_{\nu}\right]=i \hbar \delta_{\nu}^{\mu}+\frac{i \hbar}{6} J_{\nu \alpha \beta}^{\mu}(Q)\left\{\mathbf{x}^{\alpha}, \mathbf{x}^{\beta}\right\}
$$

Note that the Riemann curvature tensor can also be expresed in terms of the Jacobi curvature tensor $R^{\mu}{ }_{\alpha \nu \beta}=2 / 3\left(J^{\mu}{ }_{\nu \alpha \beta}-J^{\mu}{ }_{\beta \alpha \nu}\right)$ which means together with Eq.134 that the Jacobi curvature tensor carries exactly the same information content as the Riemann curvature tensor.

We are therefore lead to suggest the following:

An observer $A$ located at some arbitrary point $Q$ in curved spacetime uses the Heisenberg algebra with commutation relations as defined at $Q$ by the Eq.135. It should allow him to do quantum mechanics and quantum field theory in the vicinity of $Q$, namely up to the second order $\square$ in the distance $x^{\mu}$ from $Q$. This is a nontrivial ansatz for quantum theoretical momentum on curved space and we will have to compare it

\footnotetext{
${ }^{17}$ Continuing the expansion in Eqs.128 131 yields higher powers in the x's on the rhs of Eq.135 which expand the validity of Eq135 to higher orders in the distance $x^{\mu}$ from $Q$
} 
to other approaches where momentum is defined e.g. via (ordinary) Fourier transformation in an approximation or e.g. via the vanishing covariant derivative of some stress energy tensor, see e.g. 47, 48. Let us note that our definition of momentum as generating (passive) translations obeys at least the following three criteria: It is well defined how it is practically to be measured, it has the right quantum theoretical limit for vanishing curvature, and it also has the right classical limit for $\hbar \rightarrow 0$, where it is still the generator of passive translations on classical curved space, which was our definition of momentum there.

We gave an ansatz for how curvature, in an approximation, could kinematically influence the Heisenberg commutation relations of position and momentum operators. Here we will not yet study the dynamics of example systems nor will we try to express how stress-energy expectation values determine the curvature $J$. Work in this direction is in progress. Let us however discuss what it would mean for the uncertainty relations if our definition of momentum proves to be applicable:

The above commutation relations Eq.135 are of the form of our generalised Heisenberg algebras Eqs.72-74, with a nonvanishing matrix $A$, being the Jacobi tensor. However, from $J_{(\alpha \beta \gamma)}^{\mu}=0$ it is clear that these commutation relations are not precisely of the form of the examples of commutation relation for which we had Hilbert space representations. For field theoretical studies, we will therefore first have to construct Hilbert space representations for our commutation relations Eq.135, which are of course not necessarily Bargmann Fock representations. Can we already say something about the occurrence of nonzero minimal uncertainties in momenta? Note that because of $J_{\mu \mu \mu}^{\mu}=0$, nonzero minimal uncertainties in momenta will not directly appear from an equation of the type of Eq.66. However, there are in general nonvanishing elements $J^{\mu}{ }_{\nu \mu \mu}$ which have the potential to produce nonzero minimal uncertainties in the momentum $\mathbf{p}_{\nu}$ through uncertainty relations of the type $\Delta x^{\mu} \Delta p_{\nu} \geq \frac{\hbar}{12} J^{\mu}{ }_{\nu \mu \mu}\left(\Delta x^{\mu}\right)^{2}+$ rest. A detailed analysis is in progress.

Let us discuss implications on the external symmetry that could be expected from our ansatz. Our momentum operators would provide the Poincaré generators of (passive) translations and also the Poincaré generators of (passive) rotations should be contained in the Heisenberg algebra, i.e. it should be possible to express them in terms of the position and momentum operators. We had already mentioned that nonzero minimal uncertainties, in particular in energy, would e.g. make a strict distinction between positive and negative frequencies impossible, so that the propagators could reflect spontaneous particle creation and curvature induced energy (and momentum) nonconservation.

Of course, energy and momentum are not conserved on curved space since gravity is described as an intrinsic property of spacetime itself. The covariant divergence of the stress energy tensor vanishes, however, the covariant divergence decomposes into the ordinary divergence plus a term that involves the field strength $\Gamma$.

Related to this, it is on a general curved spacetime not possible to define a total 
momentum vector or e.g. a total angular momentum tensor for a collection of (even classical) particles. How would this fact be reflected in our framework? It is that the additive coalgebra structure is no longer compatible with the algebra structure of the generalised Heisenberg algebra. Let us explain this important point.

For each particle separately one can consider its position or momentum. Practically this means that the information on its position and momentum is encoded in a vector which is contained in the representation of a Heisenberg algebra of position and momentum operators. Each particle has its Heisenberg algebra acting on its states. Considering $n$ particles, or more precisely, considering $n$ - point functions, there are $n$ Heisenberg algebras acting on the tensor product of their $n$ representation spaces. So far this trivially expresses that one can speak of each particle's, or leg's, momentum, position etc separately. How is it expressed when there is also e.g. a total momentum? If the collection of the particles can be considered as a new 'total' system, it mathematically means that there is one Heisenberg algebra which not only acts on each particle representation separately but also on their tensor product representation space. E.g. the operator $\mathbf{p}_{\mu}$ that acts on one representation must therefore also act on the tensor product of two representations, namely as $\Delta \mathbf{p}_{\mu}=\mathbf{p}_{\mu} \otimes 1+1 \otimes \mathbf{p}_{\mu}$, so that the two momenta are added. The comultiplication 18 that maps the Heisenberg algebra $H$ onto its tensor square $\Delta: H \rightarrow H \otimes H$ can be iterated to map onto arbitrary high tensor powers of $H$, so that the Heisenberg algebra can act on arbitrary many legs to determine total momentum, total angular momentum etc.. For this to be consistent, the comultiplication has to be an algebra homomorphism, i.e. it should not matter whether one first applies the comultiplication and then uses the commutation relations or vice versa. While this is the case for the ordinary Heisenberg algebra on flat space, expressing that e.g. total momentum is well defined, it is not difficult to see, that the additive comultiplication is no longer consistent with our generalised commutation relations Eqs.135. This has of course to be expected since on curved space collections of particles can no longer be described as one particle with 'total' properties.

Note that even in the special case when the commutation relations are preserved by a quantum group (which itself has a consistent coproduct structure), the Heisenberg algebra is not consistent (i.e. is not a bialgebra) with the coproduct that would describe additivity. Recall also that our quantum mechanical wave functions $\phi_{r}$ as well as our fields $\phi_{\vec{r}}$ that we summed over in the path integral remain strictly commutative. The Bargmann Fock functions $\phi(\bar{\eta})$ are functions of operators and their noncommutativity is merely the noncommutativity of the generalised Heisenberg algebra and does not imply noncommutativity of the wave functions. This means in particular that, unlike other ansatzes, we do not describe particles with braid statistics, not even with the sub class of Heisenberg commutation relations that are preserved under the action of a quantum group.

\footnotetext{
${ }^{18} \mathrm{~A}$ comultiplication obeys the axioms dual to the axioms of an algebra i.e. with the direction of the arrows reversed, thus being coassociative, having a counit etc., see e.g. [2, 3]
} 
The connection between spin and statistics on a general curved, and in the ultraviolet possibly noncommutative space must be intimately related to unitarity and the action of the Poincaré generators. We will here not further discuss these questions. The aim of this section was merely to sketch a possible ansatz for generalised Poincaré operators, namely as generators of passive translations and rotations, and to show that this naturally leads to the type of generalised commutation relations that we had considered in the previous sections.

\section{$5 \quad$ Summary and Outlook}

We followed the idea that gravity puts (dynamical) lower limits to the precision to which positions or momenta can be determined, and studied whether this could lead to a natural regularisation of divergencies. Technically we made the ansatz that these lower limits of precision take the form of nonzero minimal uncertainties, where we applied the usual quantum theoretical definition of the uncertainty in an observable.

The ordinary quantum field theoretical framework does not have lower limits to the accuracy to which positions or momenta could formally be described. This feature is independent of the actual interactions since uncertainty relations are a purely 'kinematical' consequence of the Heisenberg commutation rules.

Therefore, in order to introduce nonzero minimal uncertainties in positions and momenta, we defined generalisations of the quantum theoretical framework itself, by generalising the Heisenberg algebra of positions and momenta. After developing useful algebraic techniques in the quantum mechanical case, we proceeded to the study of quantum field theory. It was crucial to realise that in quantum field theory too the Heisenberg algebra of 4-positions and 4-momenta 'sets the stage': Points e.g. in position space are points in the continuous spectra of the position operators and e.g. the scalar product of position and momentum eigenvectors defines the unitary transformation from position to momentum space. A field is an abstract vector in the representation space of the Heisenberg algebra.

Choosing the Bargmann Fock basis for the fields $\mathbb{F}^{\mathbb{g}}$ i.e. for the vectors in this representation space, we reformulated the ordinary quantum field theoretical path integral in this new basis. No longer needing or assuming a position or a momentum eigenbasis, we were then able to generalise the Heisenberg algebra. In the general case the position operators (as well as the momentum operators) no longer commute even among themselves, i.e. 'position and momentum space become noncommutative'. As a consequence 'points' in position or momentum space i.e. position or momentum eigenvectors are abandoned and instead there naturally appear nonzero minimal un-

\footnotetext{
${ }^{19}$ Recall that while it is usual practice to choose the Bargmann Fock basis in the space in which the fields take their values, we are here choosing the Bargmnn Fock basis in the space on which the fields are defined.
} 
certainties in positions and momenta.

We were led to a large class of generalisations of the Heisenberg algebra which imply these nonzero minimal uncertainties. Representations for two sub classes of these generalised Heisenberg algebras were supplied by algebraic techniques that have been developed in the field of quantum groups. We could therefor in two cases work out explicitly the Feynman rules and the example of a loop integration in scalar $\phi^{4}$ theory. In both cases we showed the regularisation of a quadratically ultraviolet divergent graph through the generalisation.

This supports the idea that the introduction of nonzero minimal uncertainties provides a new regularisation method. We do not yet have a general proof. Given representations not only of our two sub classes but also for the general Heisenberg algebras described by Eqs.72-74, it should be straightforward to work out also the correspondingly generalised quantum field theoretical framework and to check the convergence of loop integrations.

Thereby the corresponding generalisations of the framework itself are determined by sticking to the definition that the path integral is the sum over all 'fields', i.e. the sum over all vectors in the representation of the Heisenberg algebra of 4-positions and 4-momenta. It is of course not necessary that the Heisenberg commutation relations are conserved by the action of a quantum group, as it is the case in the first of the two sub classes of Heisenberg commutation relations that we worked with.

Once representations of our generalised Heisenberg algebras Eqs 72.74 are found, our framework offers many possibilities to formulate and to study completely new ansatzes for locality in the ultraviolet. As we said, one can possibly even extend the beautyful position and momentum duality from the kinematical to the dynamical level, which would relate the very small to the very large scales in a natural way.

Another important issue for further study is the fact that due to ordering ambiguities it is not straightforward to find the 'correct' generalisation of the action functional. A guiding principle for the generalisation of Lagrangians must be found on physical grounds (one may even speculate that such a principle could single out Lagrangians that have generalisations i.e. ultimately, which could be consistent with the description of gravity). In particular, through the introduction of nonzero minimal uncertainties in positions, the notions of 'pointlike' particles, of point-interaction and of locality will be generalised. In our calculations we have so far only used a simple working hypothesis for the generalised locality.

We should keep in mind that among the main criteria for the exploration of the new possibilities must be the conservation of the basic features of quantum field theory microcausality and unitarity. Also the relation between spin and statistics has to be clarified. Like in ordinary quantum field theory it is therefor crucial to understand the rôle of the (local) Poincaré algebra of translation and rotation generators. It is contained in the Heisenberg algebra and we must therefor study first the origin of the generalised commutation relations. 
To this end we discussed possible anatzes to make the origins of ultraviolet and infrared modifications of the Heisenberg algebra explicit. For the modifications that imply nonzero minimal uncertainties in positions we found motivation in the literature, coming from studies in quantum gravity and string theory where uncertainty relations of exactly our type had already appeared. We also proposed an ansatz for how large scale gravity enters into the definition of the momenta as the generators of passive translations. One then indeed straightforwardly arrives at infrared modified commutation relations of the expected form, which have the potential to produce dynamical nonzero minimal uncertainties in momenta. We explained our further programme in this direction, namely to study the local Poincaré algebra generated by momenta and angular momenta etc. which are identified as the generators of passive translations and rotations.

As we said, our framework could be considered as being simply an approach towards a new regularisation method. However, we are here not the first to suggest that new physical insight could be gained by studying the interplay of quantum theory, the measurement process and gravity, see e.g. [52]. Although quite different from previous ansatzes, also our ansatz of the use of noncommutative geometry with its nonzero minimal uncertainties can probably be interpreted in this way.

Ultimately we would expect the fully developed formalism to express a dynamical interdependence of gravity and quantum theory: On the one hand the 'local' curvature would influence the 'local' Heisenberg commutation relations. On the other hand the generalised Heisenberg algebra would influence the very concept of locality by producing modified uncertainty relations and nonzero minimal uncertainties in positions and momenta. Both, ordinary quantum theoretical commutation relations as well as ordinary curved manifolds would only exist as limiting cases and the framework could therefore probably be called being of type IV in the discussion given in [53].

We can here only offer preliminary results, however, any possibility that gravity effects could, instead of rendering quantum field theories nonrenormalisable, actually make them finite, should be worth exploring.

Acknowledgement: It is a pleasure to thank many members of our department for the stimulating atmosphere, for their interest and for their criticisms, in particular T. Brzeziński, S.B. Davis, G. Gibbons, M.B. Green, A. Kent, P.V. Landshoff, A. Macfarlane, S. Majid, M. Perry, T. Samols, A.R. Steif, J.C. Taylor and G.M.T. Watts.

\section{References}

[1] S. Weinberg, Ann. Phys. N.Y. 194, 336 (1989)

[2] M. E. Sweedler, Hopf algebras, Benjamin (1969) 
[3] E. Abe, Hopf algebras, Cambridge University Press (1980)

[4] V. G. Drinfel'd, in Proc. ICM Berkeley, AMS, Vol. 1, 798-820 (1986)

[5] L. D. Faddeev, N. Yu. Reshetikhin, L. A. Takhtajan, Alg. Anal. 1, 1, 178 (1989)

[6] S. Majid, Int. J. Mod. Phys. A. Vol. 5, No 1, 1-91 (1990)

[7] S. Majid, Foundations of Quantum Group Theory, to appear in CUP (1994)

[8] J. Wess, B. Zumino, Nucl. Phys. (Proc. Suppl.) 18 B, 302 (1991)

[9] A. Kempf, Lett. Math. Phys. 26: 1-12 (1992)

[10] A. Kempf, J. Math. Phys., Vol. 34, No.3, 969-987 (1993)

[11] A. Kempf, in Proc. XX DGM- Conference, June '91, New York, Eds. S. Catto, A. Rocha, World Scientific, 546 (1991)

[12] A. Kempf, in Proc. XIX Symposium Ahrenshoop in Wendisch-Rietz, Eds. B. Dörfel, E. Wieczorek, DESY Preprint 93-013, (1992)

[13] A. Kempf, J. Math. Phys. 35 (9) 4483-4496 (1994)

[14] A. Connes, Publ. I.H.E.S. 62, 257 (1986)

[15] A. Connes, J. Lott, Nucl. Phys. B (Proc. Suppl.), Vol. 18, 29-47 (1990)

[16] A.P. Balachandran, G. Bimonte, E. Ercolessi, G. Landi, F. Lizzi, G. Sparano, P. Teotonio-Sobrinho, Preprint ICTP: IC/94/38, DSF-T-2/94, SU-4240-567 (1994)

[17] S. Majid, Class. Quantum Grav. 5 1587-1606 (1988)

[18] S. Majid, Int. J. Mod. Phys. A, Vol 5, No. 24 4689-4696 (1990)

[19] T. Brzeziński, S. Majid, Commun. Math. Phys. 157, 591-638 (1993)

[20] A. Dimakis, F. Mueller-Hoissen, Int. J. Mod. Phys. A (Proc. Suppl.) Vol. 3A, 474-477 (1992)

[21] L. Baulieu, E. G. Floratos, Phys. Lett. B 258, 171 (1991)

[22] A. Macfarlane, J. Phys. A 22, 4581 (1989)

[23] L. Biedenharn, J. Phys. A 22, L 873 (1989)

[24] A. K. Rajagopal, V. Gupta, Mod. Phys. Lett. A, Vol. 7, No.40, 3759-3764 (1992)

[25] A. P. Isaev, Preprint JINR E2-94-38, hep-th/9402060 (1994) 
[26] S. V. Shabanov, Preprint Saclay-T93/078, hep-th/9308002 (1993)

[27] A. Hebecker, W. Weich, in Proc. XIX Symposium Ahrenshoop, Eds. B. Dörfel, E. Wieczorek, DESY Preprint 93-013, (1993)

[28] J. Schwenck, J. Wess, Phys. Lett. B 291, 273 (1992)

[29] A. Hebecker, S. Schreckenberg, J. Schwenck, W. Weich, J. Wess, Preprint MPIPh/93-45, LMU-TPW 93-17 (1993)

[30] T.H. Koornwinder, R.F. Swarttouw, Trans. AMS 333 (1992) 445

[31] W. Pusz, S. Woronowicz, Rep. Math. Phys. 27, 231 (1989)

[32] M. Chaichian, P. Kulish, J. Lukierski, Phys. Lett. B 26243 (1991)

[33] S.N.M. Ruijsenaars, Ann. of Phys. 137, 33 (1981)

[34] S. Doplicher, K. Fredenhagen, J. E. Roberts, Preprint, Dept. of Math., Univ. of Rome "La Sapienza", March 94/6 (1994)

[35] W.G. Unruh, R.M. Wald, Phys. Rev. D 29, 1047 (1984)

[36] J. Audretsch, R. Müller, Preprint KONS-RGKU-94-03, to appear in Phys. Rev. $\mathrm{D}(1994)$

[37] P. K. Townsend, Phys. Rev. D, Vol. 15, No 10 2795-2801 (1976)

[38] M-T. Jaeckel, S. Reynaud, Phys. Lett. A 185 143-148 (1994)

[39] L. J. Garay, Preprint gr-qc/9403008, Imperial/TP/93-94/20 (1994)

[40] M.B. Green, J.H. Schwarz, E. Witten, Superstring Theory, CUP (1987)

[41] D. Amati, M. Cialfaloni, G. Veneziano, Phys. Lett. B 216, 41 (1989)

[42] K. Konishi, G. Paffuti, P. Provero, Phys. Lett. B, 234, 276 (1990)

[43] M. Maggiore, Phys. Lett. B, 31983 (1993)

[44] V. Bargmann, Commun. Pure \& Appl. Math. 14, 187 (1961)

[45] A. Perelomov, Generalised coherent states and their applications, Springer (1986)

[46] P.H. Eberhard, R.R. Ross, Found. Phys. Lett. Vol.2, No.2, 127 (1989)

[47] N.D. Birrell, P.C.W. Davies, Quantum fields in curved space, CUP (1982)

[48] Les Houches Lectures '63, Eds. C. DeWitt, B. DeWitt, Gordon \& Breach (1964) 
[49] C.W. Misner, K.S. Thorne, J.A. Wheeler, Gravitation, p.285 Freeman \& Co (1973)

[50] R.P. Feynman, Quantum Electrodynamics, p.76, Benjamin (1962)

[51] R.P. Feynman, Dirac Memorial Lecture, CUP (1987)

[52] S.W. Hawking, R. Penrose, Lectures on The nature of space and time, to appear in Princeton Univ. Press (1994)

[53] C.J. Isham, Preprint Imperial/TP/93-94/1 (1994) 